\title{
Astrocytic signaling supports hippocampal-prefrontal theta synchronization and cognitive function
}

\author{
Vanessa Morais Sardinha1,2 | Sónia Guerra-Gomes ${ }^{1,2}$ | Inês Caetano ${ }^{1,2}$ | \\ Gabriela Tavares $^{1,2}$ | Manuella Martins ${ }^{1,2}$ | Joana Santos Reis ${ }^{1,2}$ | \\ Joana Sofia Correia1,2 | Andreia Teixeira-Castro1,2 | Luísa Pinto1,2 | \\ Nuno Sousa ${ }^{1,2}$ | João Filipe Oliveira ${ }^{1,2,3}$
}

${ }^{1}$ Life and Health Sciences Research Institute (ICVS), School of Medicine, University of Minho, Campus Gualtar, Braga 4710-057, Portugal

${ }^{2}$ ICVS/3B's-PT Government Associate Laboratory, Braga/Guimarães, Portugal

${ }^{3}$ DIGARC, Polytechnic Institute of Cávado and Ave, Barcelos 4750-810, Portugal

Correspondence

João Filipe Pedreira de Oliveira, Life and Health Sciences Research Institute (ICVS), School of Medicine, University of Minho, Campus de Gualtar, 4710-057 Braga, Portugal. Email: joaooliveira@med.uminho.pt

Funding information

Foundation for Science and Technology (FCT) project (PTDC/SAU-NSC/118194/

2010) to V.M.S., S.G.G., G.T., M.M., J.S.R., J.S.C., J.F.O. and fellowships (SFRH/BD/ $89714 / 2012$ to V.M.S., SFRH/BPD/97281/ 2013 to J.F.O., SFRH/BD/101298/2014 to S.G.G., IF/00328/2015 to JFO, IF/01079/ 2014 to LP); Marie Curie Fellowship FP7PEOPLE-2010-IEF 273936 and BIAL Foundation Grants 207/14 to J.F.O. and 427/14 to LP; Northern Portugal Regional Operational Programme (NORTE 2020), under the Portugal 2020 Partnership Agreement, through the European Regional Development Fund (FEDER) (NORTE-010145-FEDER-000013); FEDER funds, through the Competitiveness Factors Operational Programme (COMPETE), and National funds, through the FCT (POCl-010145-FEDER-007038)

\begin{abstract}
Astrocytes interact with neurons at the cellular level through modulation of synaptic formation, maturation, and function, but the impact of such interaction into behavior remains unclear. Here, we studied the dominant negative SNARE (dnSNARE) mouse model to dissect the role of astrocyte-derived signaling in corticolimbic circuits, with implications for cognitive processing. We found that the blockade of gliotransmitter release in astrocytes triggers a critical desynchronization of neural theta oscillations between dorsal hippocampus and prefrontal cortex. Moreover, we found a strong cognitive impairment in tasks depending on this network. Importantly, the supplementation with D-serine completely restores hippocampal-prefrontal theta synchronization and rescues the spatial memory and long-term memory of dnSNARE mice. We provide here novel evidence of long distance network modulation by astrocytes, with direct implications to cognitive function.
\end{abstract}

KEYWORDS

astrocyte, behavior, local field potential, neuronal morphology, oscillations

\section{1 | INTRODUCTION}

Brain networks display a high degree of complexity that supports behavior. This complexity relies on functional, morphological and molecular features of neurons and glial cells that integrate them. Among the glial cells, astrocytes maintain with neurons a dialogue that was shown to underlie the functional modulation of brain networks. In this dialogue, astrocytes are able to sense and process neuronal information and reply back to neurons, due to the close apposition at neuronal synapses of thin processes endowed with neurotransmitter receptors (Araque et al.,
2014; Clarke \& Barres, 2013; Khakh \& Sofroniew, 2015; Pannasch et al., 2014), to the development of complex calcium signaling in subcellular compartments (Haydon \& Nedergaard, 2015; Perea, Sur, \& Araque, 2014; Rusakov, 2015; Volterra, Liaudet, \& Savtchouk, 2014), and to the controlled release of neuroactive substances (Araque et al., 2014; Perea et al., 2014; Petrelli \& Bezzi, 2016). Particularly, the release of active molecules by astrocytes, a process named gliotransmission, has been critically appraised and extensively described (Araque et al., 2014; Petrelli \& Bezzi, 2016; Verkhratsky, Matteoli, Parpura, Mothet, \& Zorec, 2016). Gliotransmitters such as ATP, glutamate or D-serine were shown 
to modulate the strength of neighboring synapses with functional consequences to the network output. In particular, D-serine, a potent NMDAreceptor co-agonist, was related to synaptic structure and function (Bail et al., 2015; Miller, 2004). This effect was described not only for neuronderived (Balu \& Coyle, 2012; Rosenberg et al., 2013), but also for astrocyte-derived $\mathrm{D}$-serine. The latter is thought to represent the major source of D-serine (Kang et al., 2013; Martineau et al., 2013; Martineau, Galli, Baux, \& Mothet, 2008; Oliet \& Mothet, 2006; Papouin, Dunphy, Tolman, Dineley, \& Haydon, 2017; Yang et al., 2003), and is critical for the maintenance of hippocampal synaptic plasticity (Fellin et al., 2009; Han, Peng, \& Dong, 2015; Henneberger, Papouin, Oliet, \& Rusakov, 2010; Martineau et al., 2013) and for dendritic maturation of newlyborn neurons in the hippocampus (Sultan et al., 2015).

This neuron-astrocyte dialogue at the synaptic level should in turn be reflected in network outputs. In fact, a number of strategies have been employed to tackle the involvement of astrocytes on the computation of several behavioral dimensions, namely on cognition (Oliveira, Sardinha, Guerra-Gomes, Araque, \& Sousa, 2015). However, the putative involvement of astrocytes in cognitive modulation remains unclear, mostly due to the current knowledge gap between cellular interactions and behavior levels. To address this issue, we have designed an approach to assess the role of astrocyte signaling in corticolimbic circuits involved in cognitive processing. For that we analyzed the hippocampusprefrontal cortex electrophysiological synchrony and related cognitive computation in dominant negative SNARE (dnSNARE) mouse model of astrocyte-specific exocytosis impairment (Halassa et al., 2009; Pascual et al., 2005; Sultan et al., 2015). Within the hippocampus, cognitive functions rely primarily on the dorsal subregion (Fanselow \& Dong, 2010). Despite its indirect connection to the prefrontal cortex (Preston \& Eichenbaum, 2013), oscillations of the dorsal hippocampus highly synchronize with units and ensembles in the prefrontal cortex to support cognitive tasks, such as spatial learning and memory processing (Gordon, 2011; Jones \& Wilson, 2005; O'Neill, Gordon, \& Sigurdsson, 2013). Taking advantage of the specific dnSNARE transgene expression in astrocytes of the hippocampus and cortex (Pascual et al., 2005; Sultan et al., 2015), which causes a decrease of extracellular D-serine levels in those brain regions (Sultan et al., 2015), we analyzed electrophysiology fingerprints of the hippocampus-prefrontal cortex network, carried out a battery of cognitive tests and histological/molecular correlates to confirm the involvement of astrocytic signaling in cognitive function.

\section{I MATERIALS AND METHODS}

\section{$2.1 \mid$ Animals}

All experimental procedures were conducted in accordance with the guidelines for welfare of laboratory animals, as described in the Directive 2010/63/EU, and were approved by the local ethical committee (SECVS 075/2015) and national authority for animal experimentation (DGAV). The generation of dominant-negative SNARE (dnSNARE) mice of gliotransmission impairment was performed as previously described (Pascual et al., 2005). The dnSNARE mice and respective wild-type littermates were obtained by crossing two transgenic mouse lines:
GFAP-tTA, in which the expression of tetracycline transactivator (tTA) is mediated by the astrocyte-specific human glial fibrillary acidic protein (hGFAP) promoter; tetO.dnSNARE, in which the dominant-negative domain of vesicular SNARE VAMP2/synaptobrevin II, as well as the reporter enhanced green fluorescence protein (EGFP) are coexpressed under the control of the tetO promoter. Developmental expression of dnSNARE was prevented by breeding the animals in the presence of doxycycline (Dox, Sigma-Aldrich) in the drinking water $(100 \mu \mathrm{g} / \mathrm{mL}$; water bottles covered with aluminum foil to avoid light exposure), which was removed 6 weeks before the beginning of the behavior experiments. The conditional expression of the dnSNARE transgenes caused interference with the SNARE complex formation and consecutive blockade of exocytosis specifically in astrocytes (astrocytes derived from dnSNARE mice displayed a $91 \%$ reduction in the number of fusion events) (Sultan et al., 2015), impairing the vesicular release of gliotransmitters. Founders of both mice lines were kindly supplied by Prof. Philip Haydon (Tufts University, USA), and were maintained in the C57B|6/J genetic background. All mice had ad libitum access to food and water in their home cages (max 5 mice per cage) and lights were maintained on a $12 \mathrm{~h}$ light/dark cycle (lights on 8:00 A.M. to 8:00 P.M.). Male mice within ten to 12 weeks-old were used. Their genotype was confirmed by PCR: mice negative (wild-type) or positive for both transgenes (dnSNARE) were tested, while mice expressing single transgenes (GFAP-tTA or tetO.dnSNARE) were not included. Mice of both genotypes are visually indistinguishable and were kept in the housing cages mixed. Each mouse received a numbered tag which remained constant throughout the experiment and allowed to perform the complete behavior, electrophysiological, histological, and molecular analysis in a blind manner.

\section{2 | In vivo electrophysiology}

Surgical procedures, acquisition, and analysis of local field potential (LFP) signals from the medial prefrontal cortex (mPFC) and dorsal hippocampus (dHIP) were performed as previously described (Oliveira, Dias, Jacinto, Cerqueira, \& Sousa, 2013).

Mice were anesthetized with sevofluorane (4\%, SevoFlo, Abbott, USA) and the body temperature controlled and maintained at $37^{\circ} \mathrm{C}$ by a homoeothermic blanket (Stoelting, Ireland). When deeply anesthetized each animal was mounted on the stereotaxic apparatus (KOPF, USA). To avoid local pain during the surgery, Lidocaine (2\%, B. Braun, Germany) was injected subcutaneously in the area of incision. The eyes were covered with an ophthalmic cream (Duratears Z, Alcon, USA) to avoid dehydration. Experimental procedures for electrode implantation were described previously (Oliveira et al., 2013). Briefly, concentric platinum/iridium recording electrodes $(400 \mu \mathrm{m}$ shaft diameter; Science Products, Germany) were placed in the prelimbic area (PL) of the mPFC (coordinates: $1.94 \mathrm{~mm}$ anterior to bregma, $0.4 \mathrm{~mm}$ lateral to the midline, $2.5 \mathrm{~mm}$ below bregma), and in CA1 of dHIP (coordinates: $1.94 \mathrm{~mm}$ posterior to bregma, $1.2 \mathrm{~mm}$ lateral to the midline, $1.5 \mathrm{~mm}$ below bregma), according to the mouse brain atlas (Paxinos \& Franklin, 2001). A stainless-steel screw was placed above the contralateral hemisphere and used as reference electrode. 
Local field potential (LFP) signals from the MPFC and dHIP were acquired and analyzed as previously described (Oliveira et al., 2013). These signals were simultaneously amplified, filtered (5000×; $0.1-$ 300 Hz; LP511 Grass Amplifier, Natus, USA), acquired (Micro $1401 \mathrm{mkIl}, \mathrm{CED}, \mathrm{UK})$ and recorded on a personal computer running Signal Software (CED, UK). After surgery and a resting period of $20 \mathrm{~min}$, $100 \mathrm{~s}$ of local field activity were recorded at the sampling rate of $1000 \mathrm{~Hz}$. The power of PFC and dHIP regions, as well as the coherence assessment between both regions, were performed on LFP signals acquired for each mouse. Each measure was applied on $1 \mathrm{~s}$ long segments and the average of all segments was considered for statistical analysis. All LFP recordings were thoroughly inspected and those that presented significant noise corruption were excluded from further analyses. Power and coherence were calculated with custom-written MATLAB-based programs and scripts (MathWorks, USA), using the Chronux toolbox (http://www.chronux.org) (Mitra \& Pesaran, 1999), for all frequencies from 4 to $40 \mathrm{~Hz}$. For power quantification, the squared magnitude of Fourier data was evaluated, across the frequency domain for each brain region. Coherence analysis was based on multi-taper analysis of the signal magnitude for both regions. For group comparison, three frequency bands were analyzed based on previously described functional relevance: theta $(4-12 \mathrm{~Hz})$; beta $(12-20 \mathrm{~Hz})$; low gamma $(20-40 \mathrm{~Hz})$.

After the electrophysiological protocol, animals were euthanized with a lethal dose of sodium pentobarbital ( $150 \mathrm{mg} / \mathrm{kg}$, i.p). A biphasic stimulus ( $5 \mathrm{~s}, 0.7 \mathrm{~mA}$ for $\mathrm{mPFC}$, and $0.8 \mathrm{~mA}$ for $\mathrm{dHIP}$ ) was delivered to both electrodes in order to mark the local of recording. Brains were carefully removed and the left hemisphere (electrodes location) was immersed in paraformaldehyde 4\% (PFA) in PBS (0.1M, pH 7.4) for tissue fixation. One day after, it was sectioned in $50 \mu \mathrm{m}$ slices using a vibratome (Leica Biosystems, Germany) and processed with cresyl violet staining to identify the electrolytic lesion at the recording sites. Whenever at least one of the electrodes was wrongly positioned in the targeted region, mice were discarded from the analysis (about 15\% of the recordings). The right hemisphere was macrodissected and cryopreserved for molecular analysis.

\section{3 | Behavior}

Mice were tested during the light phase. Prior to experimentation, mice were handled daily for $5 \mathrm{~min}$, throughout 1 week, and habituation to testing rooms was performed $1 \mathrm{~h}$ before the beginning of each test.

\subsubsection{Morris water maze}

Morris water maze (MWM) was used to assess spatial reference memory, slightly modified from Lima et al. (2014). The test was conducted under dim light in a dark circular pool $(106 \mathrm{~cm}$ diameter) filled with water at $23^{\circ} \mathrm{C} \pm 1{ }^{\circ} \mathrm{C}$, with extrinsic visual cues (square, triangle, horizontal stripes, and cross). In order to increase the contrast to detect the mice, water was made opaque with the addition of nontoxic titanium dioxide (Sigma-Aldrich; $250 \mathrm{mg} / \mathrm{L}$ ). A circular escape platform $(10 \mathrm{~cm}$ diameter, $22 \mathrm{~cm}$ height) was placed in one of the four imaginary quadrants of the pool submerged $1 \mathrm{~cm}$ below the water surface. The 4 days of protocol consisted in a hippocampal-dependent task whose goal was to assess the ability of mice to learn the position of the hidden platform kept always in the same position. Each day, mice performed four consecutive trials (maximum of $60 \mathrm{~s}$, with a $30 \mathrm{~s}$ intertrial interval) being placed in the pool facing the maze wall and oriented to each of the extrinsic cues in random order. Whenever mice failed to reach the platform, they were manually guided to the platform for $30 \mathrm{~s}$ before being positioned at a new starting point. Escape latencies, distances swam and swim pattern were monitored and analyzed using a video camera and the EthoVision XT 11.5 software (Noldus, The Netherlands).

\subsubsection{Hole board}

The hole board $(\mathrm{HB})$ task was performed to assess spatial orientation, namely reference and working memory (Castilla-Ortega et al., 2010). Transgenic mice and WT littermates were food restricted for 4 days before the beginning of the test to achieve a body weight drop to 80$85 \%$ of their free-feeding weights and food deprivation lasted through the whole behavioral experiment. The behavioral test was performed in a hole board containing 16 equidistant holes $(6 \mathrm{~cm}$ apart, $1.5 \mathrm{~cm}$ diameter, $2.5 \mathrm{~cm}$ depth; MedAssociates, USA). The hole board was placed inside a maze with Plexiglas walls with a different cue in each wall for orientation. All mice were submitted to 3 days of habituation (1 daily session of $10 \mathrm{~min}$ ) and one food pellet was placed in each of the 16 holes to attract mice to explore holes and eat the pellets. In the following 4 days, only a fixed set of 4 holes was baited with a pellet in a pattern that remained constant ( 2 sessions per day with an intersession interval of $2 \mathrm{~h}$, each session consisting in two trials with an intertrial interval of $45 \mathrm{~s})$. Each trial had a maximum duration of $5 \mathrm{~min}$, finishing whenever the animal found all four rewards. Mice were manually placed inside the $\mathrm{HB}$ arena at one of four random starting locations. Arenas were cleaned with $10 \%$ ethanol between trials. Tests were evaluated by tracking animals trail with an infrared detection system and a computer interface. The following measures were analyzed: RM ratio (number of visits to the baited set of holes over the number of visits to all holes-provides an index for the ability of animals to discriminate between baited and unbaited holes); and WM ratio (number of rewarded visits over the number of visits to the baited set of holes-reflects the ability of the animals to avoid re-visits to baited holes during a trial).

\subsubsection{Novel object recognition}

The novel object recognition (NOR) task (Leger et al., 2013) was conducted under dim white-light illumination in a lusterless white box $(30 \times 30 \times 30 \mathrm{~cm})$. Habituation to the empty box was performed in three consecutive days for $20 \mathrm{~min}$. In the fourth day of the task animals were submitted to a training phase consisting in the exploration of two equal objects (glass bottles) for $10 \mathrm{~min}$. In the fifth day, for the novel object recognition phase, one of the familiar objects was replaced by a novel (Lego $\AA$ brick) and mice were placed in the arena and allowed to explore both for $10 \mathrm{~min}$. This trial assesses the ability to recognize a novel object $24 \mathrm{~h}$ after the first exposition, evaluating long-term recognition memory. After an interval of $1 \mathrm{~h}$ in their home cages, the novel object was displaced to the opposite side of the box and mice were allowed to 
explore this new configuration. This trial allowed the assessment of the spatial recognition memory of the subjects. Boxes were cleaned between trials and subjects with $10 \%$ ethanol. Behavior was video-recorded and analyzed using EthoVision XT 11.5 software (Noldus, The Netherlands). Exploration time of the novel or displaced objects over the total exploration time was used as measure of object preference.

\subsection{4 | Two-trial place recognition}

The two-trial place recognition (2TPR) task is based on the innate predisposition of rodents to explore novel environments. The 2TPR is a test performed in the Y-Maze arena to assess spatial recognition memory, a form of episodic-like memory in rodents (Costa-Aze, Quiedeville, Boulouard, \& Dauphin, 2012). It does not require learning of a rule being useful in particular for the study of genetic influences on the response to spatial novelty. The $\mathrm{Y}$-maze consisted in an apparatus made of white Plexiglas with three identical arms (33.2 $\mathrm{L} \times 7 \mathrm{~W} \times 15 \mathrm{~cm} \mathrm{H}$ ) which were randomly designated start (S), familiar (F), and novel (N) arms. Visual cues were placed at the end of each arm of the maze. In the first trial, mice were placed in the start arm and allowed to explore only two arms ( $S$ and F) for $5 \mathrm{~min}$. Mice were replaced in their home cages for a period of $30 \mathrm{~s}$, and then they were placed in the start arm of the maze and allowed to explore the three arms for 2 min for memory retrieval. The maze was cleaned with $10 \%$ ethanol between trials and subjects, and the test was performed in a dim light room. Mice trail was videorecorded and analyzed using EthoVision XT 11.5 software (Noldus, Netherlands). The percentage of time spent in the distal third of the novel arm was considered a measure of spatial recognition memory.

\section{$2.4 \mid$ Molecular analysis}

\subsubsection{Macrodissection, RNA isolation, cDNA synthesis, and real-time PCR analysis}

In order to assess the transcription levels of the dnSNARE and EGFP transgenes, relative mRNA levels of both genes were quantified by RTPCR. Animals were first anesthetized with a mixture of ketamine (75 mg/kg, i.p.; Imalgene 1000, Merial, EUA) and medetomidine (1 mg/ kg, i.p.; Dorbene Vet, Pfizer, EUA) and transcardially perfused with $0.9 \%$ saline. Brains were carefully removed and macrodissected, and tissue samples were stored at $-80^{\circ} \mathrm{C}$ until further analysis. To avoid experimenter-dependent bias, all brains were macrodissected by a single investigator.

Total RNA was isolated from macrodissected tissue of the prefrontal cortex of dnSNARE mice ( $n=15$ ), using the Direct-zol RNA miniPrep kit (Zymo Research, USA), according to manufacturer's instructions. Briefly, tissue was mechanically homogenized with a syringe and 20G needle using the NZYol reagent (NZYTech, Portugal).

Total RNA (500 ng) was reverse-transcribed using qScript ${ }^{\mathrm{TM}}$ cDNA SuperMix (Quanta Biosciences, USA). The following primers were designed using PRIMER-BLAST (NCBI, http://www.ncbi.nlm.nih.gov/ tools/primer-blast/) and used for expression quantification: EGFP (F 5'CCCGACAACCACTACCTGAG-3'; R 5'-ACTTTGACCATCAGAGGACATT-3'); dnSNARE (F 5'-TACCAGTAACAGGAGACTGC-3'; R 5'-
ACTTTGACCATCAGAGGACATT-3'). Quantifications were performed using the Fast Real-Time PCR System (Applied Biosystems, USA) and $5 \times$ HOT FIREPol ${ }^{\circledR}$ EvaGreen $^{\circledR}$ qPCR Mix Plus, ROX (Solis Biodyne, Estonia). Target gene expression levels were normalized against the housekeeping gene $18 \mathrm{~S}$ rRNA and the relative expression was calculated using the $\Delta \Delta \mathrm{Ct}$ method.

\subsubsection{Western blot analysis}

In order to screen the transgenic protein levels in the brain regions analyzed in the behavior and electrophysiology experiments, relative EGFP levels were quantified by western-blot. Brain samples containing the hippocampus were lysed in cold HEPES-buffered sucrose $(0.32 \mathrm{M}$ sucrose, 4 mM HEPES, pH 7.4) with $1 \%$ Nonidet-P40, 0.5\% SDS, and a mixture of protease inhibitors (cOmplete, EDTA-free, Roche, Switzerland). Then, samples were sonicated for $10 \mathrm{~s}$ and centrifuged at $10,000 \mathrm{rpm}$ during $25 \mathrm{~min}$ at $4^{\circ} \mathrm{C}$. The supernatant was collected and protein concentrations were determined using the Bradford protein assay (Bio-Rad, USA). Total lysates were denatured in $2 \times$ Laemmli buffer (Bio-Rad, USA) by heating for $5 \mathrm{~min}$ at $100^{\circ} \mathrm{C}$. Each sample was centrifuged during $10 \mathrm{~s}$ before loading. Equal protein amounts $(50 \mu \mathrm{g})$ were loaded into SDS-PAGE (10\%) gels and then transferred to a nitrocellulose membrane (Trans-blot Turbo Kit, Bio-Rad, USA). Membranes were blocked in $5 \%$ dry milk/TBS ( $1 \mathrm{~h}$ ) before incubation overnight at $4^{\circ} \mathrm{C}$ with the primary antibodies: mouse anti- $\alpha$-tubulin (1:500, DSHB, USA); goat anti-GFP (RRID: AB_305643; 1:2000, Abcam, UK). After washing with TBS-T, membranes were incubated with secondary antibodies: anti-mouse HRP (RRID: AB_11125547; 1:15,000; Bio-Rad, USA) and antigoat HRP (1:5000, Bio-Rad, USA), respectively. Detection of the chemiluminescent signal was performed with the Clarity Western ECL substrate kit (Bio-Rad, USA) using a gel blot imaging system (Chemidoc, Bio-Rad, USA). Band quantification was assessed using the Image Lab software (Bio-Rad, USA), and all the samples were normalized according to the loading control ( $\alpha$-tubulin). Given the difficulty of quantifying dnSNARE protein levels in brain tissue (available antibodies detect similarly both the truncated and endogenous forms of synaptobrevin 2), and the fact that the EGFP levels observed are highly correlated with the EGFP mRNA levels, which in turn directly correlate with the dnSNARE levels, the quantification of GFP by western blot was used throughout to screen the transgene expression levels in all mice tested. To confirm the induction of expression of dnSNARE transgenes after Dox removal from the diet, groups of 2 dnSNARE mice were sacrificed at different timepoints (on Dox, 1, 2, 3, 4, and 8 weeks after Dox removal). Additionally, 2 wild-type littermates were sacrificed for negative control. Protein extracts were obtained from whole-brain lysates. To quantify the relative levels of GFP across brain regions and across animals, the brains of 13 dnSNARE mice were macrodissected 6 weeks after Dox removal, and each brain region was processed independently by western blot as described above.

\subsection{3 | Immunofluorescence analysis}

Brain tissue of the tested animals was stained by immunofluorescence to visualize the expression of transgene reporters, to study the co-expression with astrocytic and neuronal markers, to allow the 
3D reconstruction of astrocytes and assessment of astrocytic densities. Mice were deeply anaesthetized with the ketamine and medetomidine mix, and readily perfused transcardially with $4 \%$ PFA. Brains were carefully removed and immersed during $48 \mathrm{~h}$ in $4 \%$ PFA. After impregnation with $30 \%$ sucrose PBS solution (at $4{ }^{\circ} \mathrm{C}$, until sinking), brains were frozen by immersion in isopentane, cooled with liquid nitrogen in Neg-50 medium (ThermoFisher Scientific, USA) and stored at $-20^{\circ} \mathrm{C}$. The immunofluorescence procedures were performed in coronal brain sections ( $20 \mu \mathrm{m}$ thick) obtained by means of a cryostat (Leica, Germany). The double staining protocol started with three washes with PBS followed by a permeabilization with $0.3 \% \mathrm{v} / \mathrm{v}$ Triton X-100 in PBS. Sections were washed over again and submitted to an antigen retrieval step, with citrate buffer (10 mM, pH 6.0, Sigma-Aldrich) during 20 min at $100 \mathrm{~W}$ microwave potency. Once cooled, slices were rinsed in PBS and incubated with $10 \%$ fetal bovine serum (FBS) in PBS blocking solution for $30 \mathrm{~min}$ at room temperature (RT), followed by the overnight incubation, at $4^{\circ} \mathrm{C}$, with combinations of the primary antibody goat polyclonal anti-GFP (RRID: AB_305643; 1:300, Abcam, UK) and one of the following: rabbit polyclonal anti-GFAP (RRID: AB_10013482; 1:200, DakoCytomation, Denmark), or rabbit polyclonal anti-S100 $\beta$ (1:200, DakoCytomation, Denmark) for staining of astrocytes; or mouse polyclonal anti-NeuN (RRID: AB_10807945; 1:100, Millipore, Germany) for staining of mature neurons; all prepared in PBS with $0.3 \%$ Triton X-100 and $4 \%$ fetal bovine serum (FBS). On the next morning tissue sections were rinsed in PBS and then incubated with the respective speciesspecific secondary antibodies: Alexa Fluor ${ }^{\circledR} 488$ donkey antigoat, Alexa Fluor ${ }^{\circledR} 594$ donkey antirabbit and Alexa Fluor ${ }^{\circledR} 594$ donkey antimouse (1:1000, ThermoFisher Scientific, USA) in PBS with 4\% FBS, during $2 \mathrm{~h}$ at room temperature. After rinsing the brain slices with PBS, the nucleic acids were indiscriminately labeled by 10 min incubation with DAPI (RRID: AB_2307445; 1:1000, Invitrogen, USA) in the dark. After a final series of rinses in PBS, slides were mounted using Immu-mount (ThermoFisher Scientific, USA) mounting media and evaluated through confocal microscopy imaging (FV1000, Olympus, Japan) and FIJI open source software (http://fiji.sc/Fiji).

\section{5 | 3D-reconstruction of neurons}

All procedures employed to assess the morphology of neurons were previously described (Lima et al., 2014). In order to assess dendritic morphology of hippocampal neurons a set of animals ( $n=5 \mathrm{WT}$ and $n=5$ dnSNARE) were perfused with $0.9 \%$ saline under deep anesthesia (ketamine and medetomidine mix) and were processed for GolgiCox staining. Briefly, brains were removed and immersed in Golgi-Cox solution (1:1 solution of $5 \%$ potassium dichromate and $5 \%$ mercuric chloride diluted $4: 10$ with $5 \%$ potassium chromate) for 14 days; transferred to a $30 \%$ sucrose solution (minimum 3 days) and sectioned by means of a vibratome. Coronal sections ( $200 \mu \mathrm{m}$ thick) were collected in $6 \%$ sucrose and blotted dry onto gelatin-coated microscope slides and subsequently alkalinized in $18.7 \%$ ammonia, developed in Dektol (Kodak, USA), fixed in Kodak Rapid Fix, dehydrated and xylene cleared, mounted, and coverslipped. All incubation steps were performed in a dark room.
Three-dimensional (3D) reconstruction of Golgi-Cox impregnated neurons was performed in the CA1 subregion of the dorsal hippocampus following the mouse brain atlas (Paxinos \& Franklin 2001). CA1 pyramidal neurons were readily identified by their characteristic triangular soma-shape, apical dendrites extending toward the stratum radiatum. Neurons were selected for reconstruction following these criteria: (i) identification of soma within the pyramidal layer of CA1 (ii) full impregnation along the entire length of the dendritic tree; (iii) no morphological changes attributable to incomplete dendritic impregnation of Golgi-Cox staining or truncated branches. To avoid selection bias, brain slices containing the region of interest were randomly searched and the first six neurons from each animal were reconstructed from at least three brain slices (containing both hemispheres). The dendritic reconstruction was performed by using a motorized microscope controlled by the Neurolucida software (MBF Bioscience, USA) under $100 \times$ magnification. The analyzed dendritic features were: total length, number of endings and nodes and Sholl analysis (number of dendrite intersections at radial intervals of $20 \mu \mathrm{m}$ ). Dendritic spine densities were also assessed in randomly selected $30 \mu \mathrm{m}$ segments of three proximal and three distal dendritic branches per neuron, and were categorized in four types (thin, mushroom, wide, and ramified). The features of both reconstructed neurons and spines were quantified using NeuroExplorer software (MBF Bioscience, USA).

\section{6 | D-Serine administrations}

In order to rescue the functional impairments displayed by dnSNARE mice, D-serine was administered to mice of both genotypes during electrophysiology and behavior experiments (Sigma-Aldrich, USA; $1 \mathrm{~g} / \mathrm{kg}$ of body weight, $10 \mathrm{~mL} / \mathrm{kg}$ of body weight in saline, i.p.). This administration was described to increase the intracerebral levels of $\mathrm{D}$-serine and rescue functional deficits in different contexts and independent laboratories (Guercio et al., 2014; Han et al., 2015; Takata et al., 2011). Details are provided as Supporting Information.

For the electrophysiological experiments, surgical procedures and electrode placement were performed as described above. After the surgery and resting period, 15 periods of $100 \mathrm{~s}$ of local field activity were recorded each $5 \mathrm{~min}$. D-Serine was administered i.p. after the third recording, and the effect of $D$-serine was observed during the next 12 recordings (60 $\mathrm{min}$ ). The first three recordings represent the basal activity, while the 12 following recordings represent the longitudinal effect of $D$-serine administration. The electrode positions were verified as described above.

For the behavior experiments, either D-serine or saline were administered to an independent set of mice 20 min before the first trial on each day in the MWM task, or 20 min before the training session in the NOR task. Despite the i.p. injection, mice behaved normally and no signs of distress or potential drug side effects were observed, therefore no animal was excluded for those reasons.

\section{7 | Statistical analysis}

Results are presented throughout as mean \pm SEM (standard error of the mean) and the statistical significance of the comparisons for 
each statistical test was set with a confidence interval of at least 95\%. All data sets passed the normality tests for Gaussian distributions (D'Agostino and Pearson for $n>7$; Kolmogorov-Smirnov for $n=5-6)$, therefore parametric tests were applied. Two-way analysis of variance repeated measures (ANOVA) and Sidak post hoc tests were used to analyze the performance in the MWM, HB (factors: genotype and test day); and Sholl analysis data for neuronal 3D reconstructions (factors: genotype and radial distance from soma). Two-way ANOVA and Fisher's LSD test were applied to analyze the 2TPR, spines distribution and effects of D-serine. Oneway ANOVA was used to compare data between three groups (WT, dnSNARE, and IGFP). Pearson coefficients were calculated to assess correlations between: mRNA relative levels of dnSNARE and EGFP transgenes; between behavior performance and GFP levels or electrophysiological activity. In the remaining experiments, unpaired two-sided $t$ tests were applied to compare data between the two groups: WT and dnSNARE. Statistical analysis was performed using the GraphPad Prism 6 (GraphPad Software, USA).

\section{3 | RESULTS}

\section{1 dnSNARE transgenes are highly expressed in the hippocampus and prefrontal cortex and display intersubject variability}

We employed the dnSNARE mouse model (Pascual et al., 2005) to study the role of gliotransmission in complex cognitive processing, which allowed the inducible blockade of the vesicular release by exocytosis selectively from astrocytes (Fellin et al., 2009; Sultan et al., 2015), preventing developmental effects that could mask potential behavior implications. This strategy selectively perturbs exocytosis-dependent gliotransmission (Sultan et al., 2015; Zhang et al., 2004), thus allowing to dissect its implications for the neuron-astrocyte network and behavior. Independent studies showed that dnSNARE expression in astrocytes is accompanied by reduction of available gliotransmitters in cell culture (glutamate) (Zhang et al., 2004), in brain slices of hippocampus (ATP) (Cao et al., 2013) or cortex (D-serine and ATP) (Cao et al., 2013; Pankratov \& Lalo, 2015), or in vivo in the hippocampus (D-serine) (Sultan et al., 2015). In spite of being used in different labs in the past decade (Oliveira et al., 2015), the validity of this mouse model was challenged (Fujita et al., 2014). Nevertheless, we and others have recently validated this model for the study of signaling dependent on astrocyte exocytosis (Pankratov \& Lalo, 2015; Papouin, et al. 2017; Sultan et al., 2015; Wagner et al., 2017). In our lab, we firstly performed complementary sets of experiments to validate the co-expression of dnSNARE and reporter transgenes, the inducible control, the astrocyte specificity and the level of transgene expression among brain regions and subjects.

Since the dnSNARE transgene corresponds to the cytosolic portion of endogenous synaptobrevin II, the available antibodies target both proteins indiscriminately. Therefore, we assessed the co-expression of dnSNARE and enhanced green fluorescent protein (EGFP) reporter transgenes in order to use the EGFP as a reporter to screen the expression levels in all mice tested. The quantification of the relative expression of dnSNARE and EGFP mRNA by RT-PCR in one set of dnSNARE mice showed a direct correlation between the amount of transcripts of both mRNAs (Figure 1a, top), meaning that the transcription of both transgenes should follow similar regulatory mechanisms, which matches with the described elevated rate of co-localization (Halassa et al., 2009). Additionally, the relative quantification of GFP levels by western blot (using anti-GFP antibody) in the same mice showed that the relative levels of protein and mRNA follow a direct correlation (Figure 1a, bottom). Altogether, this set of data indicates that mice that express higher levels of dnSNARE, also express higher levels of EGFP mRNA, which in turn translates into increased EGFP levels in those mice. Thus, from this moment on, GFP staining or relative levels, will be used as a correlate of dnSNARE expression.

The removal of doxycycline from the mice diet triggers the expression of dnSNARE transgenes, as measured by the GFP levels of animals sacrificed at different timepoints, reaching a maximum at weeks 3-4, that remains stable in time, and is completely absent in wild-type littermates (Figure 1b); these results are in accordance with previous observations using doxycycline-controlled systems (Halassa et al., 2009; Nakashiba, Young, McHugh, Buhl, \& Tonegawa, 2008). The expression of transgenes occurred throughout the brain, displaying a typical mosaic-like distribution (Figure 1d-i) (Florian, Vecsey, Halassa, Haydon, \& Abel, 2011; Sultan et al., 2015). We consistently verified the astrocyte specificity of transgene expression, by performing immunofluorescence staining of brain slices containing the hippocampus and prefrontal cortex (regions of critical interest for this study (Gordon, 2011; Hoffmann, Cicchese, \& Berry, 2015; Lima et al., 2014; Oliveira et al., 2013; O'Neill et al., 2013)), using specific antibodies to target GFP and astrocytic or neuronal markers. GFP-labeling revealed only structures with the characteristic bushy astrocytic morphology (Florian et al., 2011; Khakh \& Sofroniew, 2015; Pascual et al., 2005). These structures were regularly positive for the astrocyte-specific marker glial fibrillary acidic protein (GFAP) (Khakh \& Sofroniew, 2015) both in the CA1 region of the dorsal hippocampus (dHIP) and in the medial prefrontal cortex (mPFC), in line with previous reports (Florian et al., 2011; Halassa et al., 2009) (Figure 1d-e). GFP was also co-localized with the astrocyte cytosolic marker S100 (Khakh \& Sofroniew, 2015) in both brain regions (Figure $1 \mathrm{f}-\mathrm{g}$ ). Together, the typical GFP-arborization and the double immunostainings indicate that the transgenes were expressed by astrocytes of both populations. Moreover, the numbers of $59.9 \pm 4.8 \%$ of GFAP-immunostained astrocytes co-expressed GFP in the dorsal hippocampus ( $n=4$ mice), as previously described (Sultan et al., 2015). Additionally, to support astrocyte specificity and to exclude neuronal expression of dnSNARE transgenes, we performed double immunostainings against GFP and NeuN (neuron specific) in the same brain regions. The detailed observation of images of each confocal section excluded staining overlap between GFP and NeuN both at the dHIP and mPFC (Figure 1h-i). Furthermore, we performed doublestainings including EGFP and two additional neuronal markers: $\beta$ III tubulin that stains neurites, and calbindin that is found in the cytosol (Supporting Information Figure S1). One clearly observes that neurites 

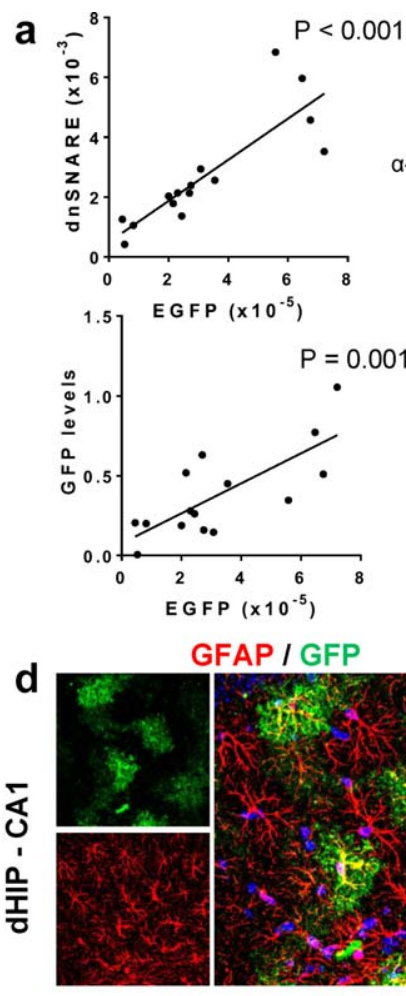

GFAP / GFP

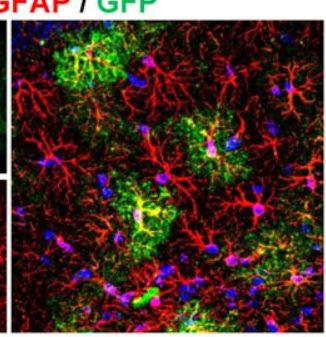

e

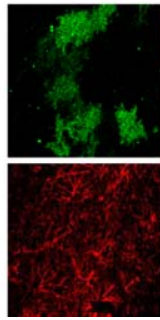

b
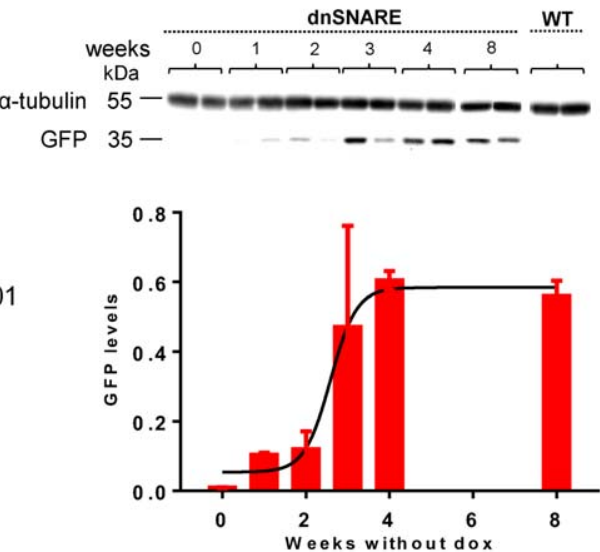

f

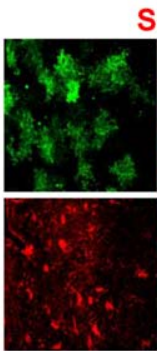

S100 $\beta$ / GFP

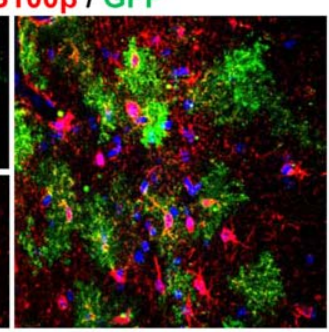

g

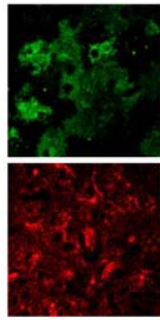

C

$\mathrm{kDa}$
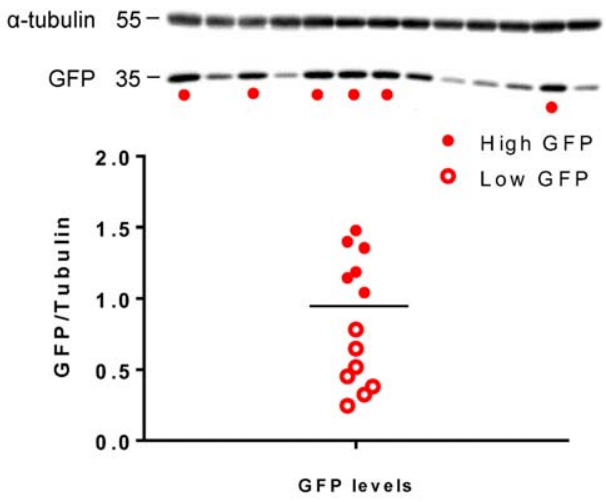

NeuN / GFP
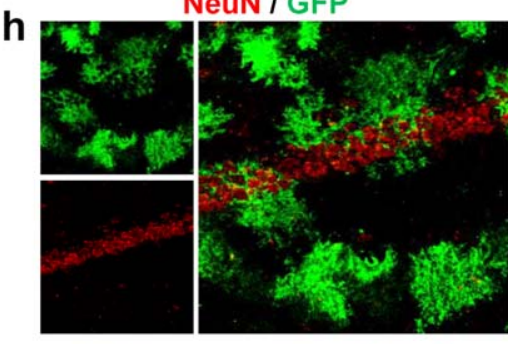

i
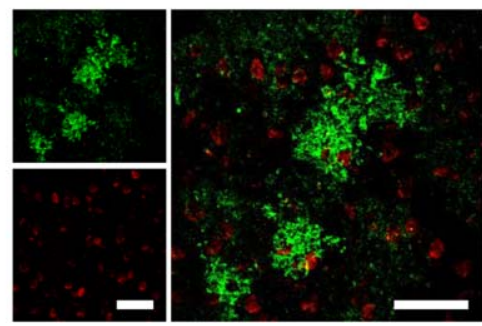

FIGURE 1 GFP reporter is a good readout of dnSNARE transgene expression and intersubject variability. (a) Pearson's correlations between relative expression levels of EGFP mRNA and dnSNARE mRNA (top), or GFP protein levels (bottom), measured in the same set of dnSNARE mice ( $n=15)$; (b) doxycycline (dox) removal from the mice diet triggers transgene expression specifically in dnSNARE mice, as assessed by the quantification of GFP ( $35 \mathrm{kDa}$ ) expression on the hippocampus of dnSNARE mice by western blot analysis at 0, 1, 2, 3, 4, and 8 weeks after dox removal from drinking water. Tubulin $(55 \mathrm{kDa})$ was used as control $(n=2 /$ timepoint); wild-type (WT) mice were used as negative controls; data plotted as mean \pm SEM. (c) Quantification of the transgene reporter GFP from the hippocampus of dnSNARE mice $(n=13)$ was performed by western blot analysis (35 kDa), relatively to tubulin $(55 \mathrm{kDa})$ for each set of animals after behavioral and electrophysiological assessment (the mean value of GFP expression, black dash, was used as criteria to separate two clusters of animals in each set: the high and low "expressors" of the transgenes; (d-e) Confocal micrographs illustrating co-expression of GFP reporter transgenes (green) with GFAP (red) in the CA1 of the dorsal hippocampus ( $D$, dHIP, stratum radiatum) and medial prefrontal cortex (E, mPFC, layers III-V) of dnSNARE mice; ( $\mathrm{f}-\mathrm{g}$ ) Confocal micrographs illustrating co-expression of GFP reporter transgenes (green) with S100 $\beta$ (red) in the dorsal CA1 (f, stratum radiatum) and mPFC (G, layers III-V) of dnSNARE mice; (h-i) confocal micrographs illustrating double staining of GFP reporter transgenes (green) and NeuN (red) in the dorsal CA1 (h, oriens, pyramidal and radiatum layers), and mPFC (i, layer V) of dnSNARE mice. DAPI staining, blue. Scale bars $=50 \mu \mathrm{m}$ [Color figure can be viewed at wileyonlinelibrary.com]

that originate in the pyramidal layer and spread across the radiatum layer are devoid of EGFP staining, which remains restricted to the bushy-like astrocyte structure. In the detail of a single confocal plane presented, three neurites clearly cross the astrocyte territory independently (Supporting Information Figure S1a,b). Regarding the calbindin double staining, we also failed to find co-localization of staining in the same cellular structures (Supporting Information Figure S1c,d). Since EGFP is also found in the cytosol, by using this combination, we definitely excluded the expression of transgenes in neurons. Absence of co-staining in microglia, NG2-positive cells and oligondendrocytes, also ruled out co-expression in these cells (Fellin et al., 2009; Sultan et al., 2015). It is noteworthy, that our observations were extremely consistent across brain areas, and across experimental sets ( $n=5$ mice screened per group), confirming the specific expression of dnSNARE transgenes in astrocytes.

In order to characterize the regional distribution of dnSNARE transgenes, we quantified the relative GFP levels across several brain regions, macrodissected from dnSNARE mice ( $n=13$; Supporting Information Figure S2). Interestingly, we observed a variable expression of transgenes across animals, which was maintained between brain 
a
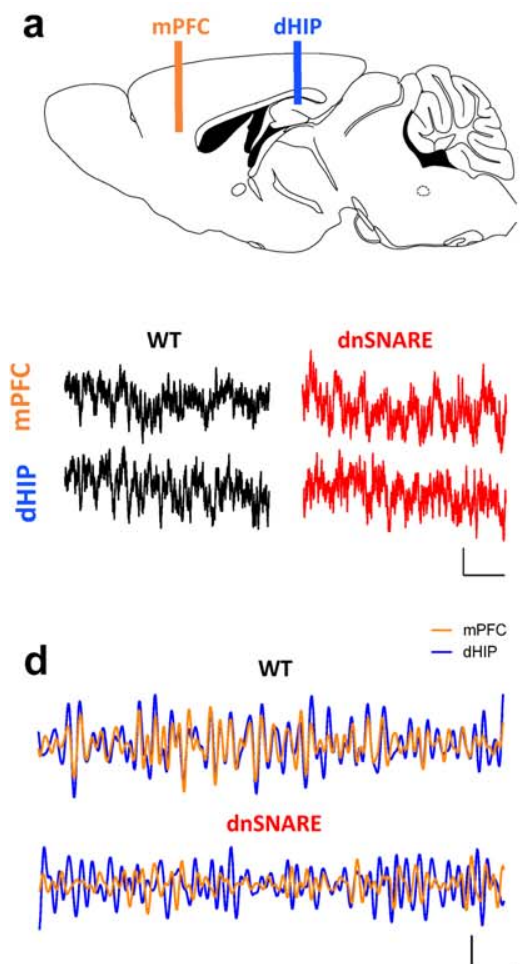

b

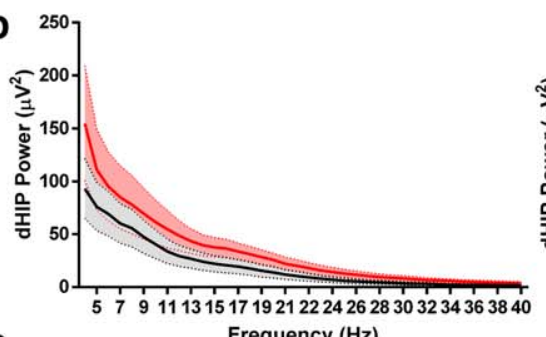

C

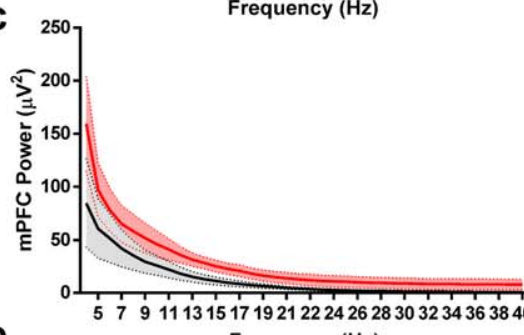

e

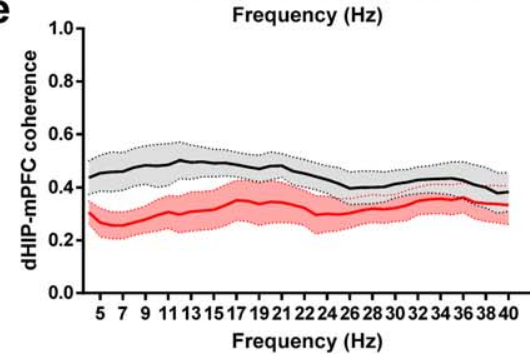

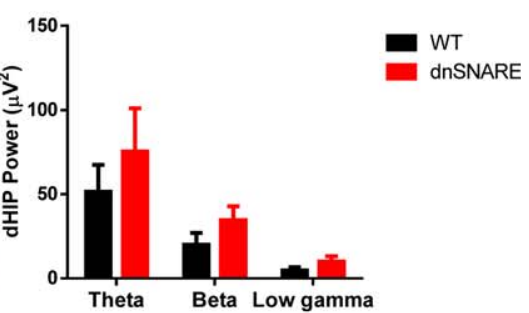
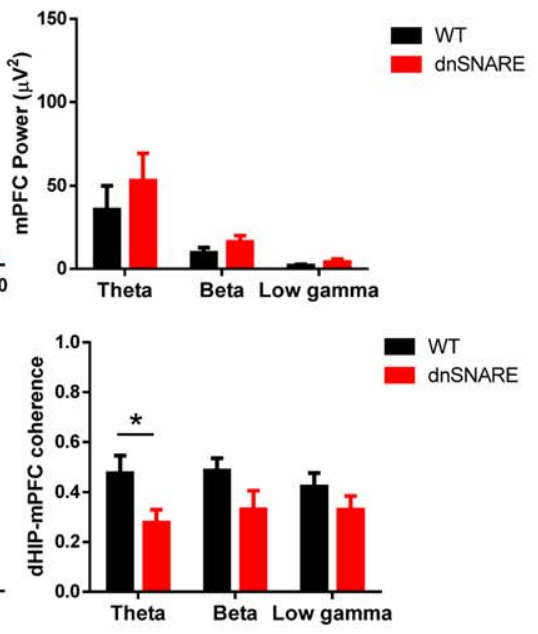

FIGURE 2 Gliotransmission impairment compromises theta coherence in the hippocampal-prefrontal network in dnSNARE mice. (a) Scheme depicting the electrode position for recording of local field potentials (LFP) in the medial prefrontal cortex (mPFC, orange) and CA1 region of dorsal hippocampus (dHIP, blue; top); representative LFP traces recorded from the mPFC and dHIP of WT and dnSNARE mice (bottom; scale bars: $100 \mu \mathrm{V}, 1 \mathrm{~s}$ ); (b-c) LFP power analysis between WT and dnSNARE; left, power spectra for dHIP (b) and mPFC (c); right, power analysis by frequency bands (theta: 4-12 Hz; beta: 12-20 Hz; low gamma: 20-40 Hz); (d) Overlap of representative theta filtered LFP traces of mPFC and dHIP, recorded from WT and dnSNARE mice (scale bars: $50 \mu \mathrm{V}, 500 \mathrm{~ms}$ ); (e) analysis of synchrony between dHIP and mPFC with dHIP-mPFC coherence spectra (left) and measured by frequency bands (right). WT mice are represented in black and dnSNARE mice in red lines and bars. Data plotted as mean \pm SEM. Two-way ANOVA, Fisher's LSD tests, ${ }^{*} p<.05$ ( $n=8-9$ per group) [Color figure can be viewed at wileyonlinelibrary.com]

regions, meaning that mice that presented high levels of expression in the hippocampus also displayed high expression in other brain regions, while the opposite was also true. The distinct levels of transgene expression observed among different animals may be due to the regulation of gene expression upstream and/or coupled with transcription. Epigenetic regulation may vary among different animals determining chromatin availability (e.g., DNA methylation, histone acetylation), which may impact directly in transgene expression both at the mRNA and protein levels. Since the expression of truncated synaptobrevin II (dnSNARE) rules the level of exocytosis blockade, one shall expect that only mice displaying high levels of dnSNARE would present an effective impairment in functions dependent on exocytotic release from astrocytes. Therefore, we divided mice into two groups, based on the relative expression of GFP: low and high transgene "expressors" (Figure 1c). Since we were interested in hippocampal-dependent behaviors, we quantified the relative GFP levels in the hippocampus of every mouse that carried both tTA and tetO transgenes (double-transgenic). This quantification was repeated for each animal set, and only those mice who expressed higher levels of GFP transgenes than the group mean were included in the dnSNARE group, being compared to their wild-type (WT) littermates throughout. Since this analysis was performed after the sacrifice, the experimenters were blind both to the animal genotype and to its relative level of transgene expression. The data including the low GFP "expressors" will be plotted as Supporting Information Figures for the verification of transgene level-dependent loss of function.

\section{2 | Astrocyte signaling is crucial for hippocampal- prefrontal theta synchronization}

Knowing that the release of gliotransmitters from astrocytes was previously shown to modulate synaptic communication in the dHIP and PFC (Araque et al., 2014; Perea et al., 2014; Petrelli \& Bezzi, 2016), one might expect that this cellular modulation might impact the function of these circuits. To address this idea, we characterized the electrophysiological fingerprints of this network in vivo, by analyzing local field potentials (LFPs; oscillations that result from coordinated rhythmic activity of neuronal populations) recorded simultaneously from the dHIP and mPFC of a set of dnSNARE mice and wild-type (WT) littermates (Figure 2a and Supporting Information Figure S3). The individual analysis of the LFP power on the frequency domain gives an estimate of the amplitude of network activity for a given frequency in the basal 
condition. The LFP power measured in WT and dnSNARE mice was similar both in the dHIP and mPFC at theta, beta and low gamma frequencies (Figure 2b,c). Moreover, the total power recorded was similar between genotypes (Supporting Information Figure S4a). The analysis of the distribution of power across frequency bands revealed that theta oscillations represent most the activity recorded in the dHIP and mPFC, however dnSNARE mice display similar levels of relative power for the different frequency bands (Supporting Information Figure S4b). These results, indicate equivalent energy of the neuronal oscillations at the given frequencies within each region, which is in line with previous observations of electroencephalogram data (Fellin et al., 2009). The analysis by specific frequency bands, defined by their functional relevance (Gordon, 2011; Oliveira et al., 2013), confirmed the power similarities between both genotypes. Next, we addressed the coherence between the LFPs recorded from the dHIP and mPFC, as a measure of phase and amplitude synchronization between these regions. Interestingly, dnSNARE mice displayed decreased levels of dHIP-mPFC coherence in the lower frequencies, visible by the superposition of thetafiltered LFP traces (Figure 2d), which were significantly different form their WT counterparts for the theta frequency band (Figure 2e). The level of decrease of dHIP-mPFC theta coherence found in dnSNARE mice is in line with previous reports from us and others linking this synchrony measure to comparable cognitive deficits (Benchenane et al., 2010; Oliveira et al., 2013; Sigurdsson, Stark, Karayiorgou, Gogos, \& Gordon, 2010). Next, we took advantage of the variable transgene expression levels observed between subjects to verify whether the neuronal activity was modulated by the level of transgene expression. Indeed, double-transgenic mice that display low levels of expression display similar electrophysiological activity to controls, being significantly different from strongly expressing-dnSNARE mice (Supporting Information Figure S5a,b). It is noteworthy that the levels of expression in double-transgenic mice strongly correlate with dHIP-mPFC theta, but not beta nor low gamma coherence (Supporting Information Figure S5c-e). Together, these results suggest that astrocyte signaling is required for maintenance of a basal theta synchronization between hippocampus and prefrontal cortex, which might be important for cognitive performance.

\section{3 | dnSNARE mice display impaired cognitive function}

Based on the available evidence linking dHIP-mPFC correct dialogue and cognitive processing (Anderson, Rajagovindan, Ghacibeh, Meador, \& Ding, 2009; Benchenane et al., 2010; Hoffmann et al., 2015; Jones \& Wilson, 2005; Oliveira et al., 2013; O'Neill et al., 2013; Siapas, Lubenov, \& Wilson, 2005; Zhan, 2015), we hypothesized that the observed loss of dHIP-mPFC synchronization might underlie a cognitive loss in mice lacking astrocyte signaling. To address this idea, independent sets of mice were submitted to tasks that assess spatial learning and longterm memory consolidation (highly dependent on the interaction between the dHIP and mPFC), using different motivations (e.g., exploratory drive, or hunger) and different environments (e.g., water-free arenas) (Figure 3).
The Morris water maze (MWM) task was performed to assess reference memory (RM) (Figure 3a-c) (Lima et al., 2014). In this task, WT animals learnt to follow the external cues to reach the platform as confirmed by the decreasing latencies and distances swam during the trials, while dnSNARE mice needed to swim longer to reach the same platform, especially in the last days of the task (Figure 3a). In accordance, dnSNARE mice failed more often to reach the platform within the $60 \mathrm{~s}$ of each trial, and whenever they reached it, they employed rather random searching and scanning strategies (Graziano, Petrosini, \& Bartoletti, 2003) indicating a poor spatial orientation (Figure 3b,c). To confirm these results, RM was additionally tested in the hole board (HB) task (Castilla-Ortega et al., 2010), which is performed in a different environment, using food deprivation as motivation to learn the location of hidden food pellets with the help of spatial cues. In this task, WT mice learnt to perform the task, as indicated by the increasing RM ratio measured along the days (Figure $3 \mathrm{~d}$ ). Also in this case, dnSNARE mice with impaired gliotransmission faced difficulties to discriminate between baited and unbaited holes, supporting an impairment in RM. Curiously, the calculation of working memory ratios (more dependent on the function of the prefrontal cortex) (Castilla-Ortega et al., 2010; Lima et al., 2014) also pointed out an impairment of the function in the dnSNARE mice, namely in the first test days. Furthermore, the novel object recognition (NOR) test was used to assess long-term memory and spatial recognition memory driven by novelty exploration (Leger et al., 2013), processes critically dependent on hippocampal and cortical networks. In this test, WT mice dedicated higher percentages of time exploring the novel object, which was not the case of the dnSNARE mice (Figure 3e). Regarding the time spent exploring the object displaced after a short period $(1 \mathrm{~h})$, although WT mice spent more time in the displaced object than dnSNARE mice, this difference was not statistically significant. These observations support a specific impairment of long-term recognition memory that matches with the hippocampal deficits observed in the previous tasks. Finally, we examined the spatial recognition memory of WT and dnSNARE mice using the two-trial place recognition task, in which animals were expected to preferentially explore a novel arm marked by spatial cues, after a short delay. This configuration allowed the assessment of both dHIP and mPFC functions (Costa-Aze et al., 2012). The analysis of percentage of time spent in the distal quadrants showed that, as expected, WT mice explore significantly more the novel arm, which was not the case of their dnSNARE littermates (Figure 3f), which reveals deficits on place recognition memory in the latter. Together, these results indicate a striking cognitive deficit, namely on functions that rely on the dHIP and its interaction with the mPFC. Double-transgenic mice that display low levels of expression perform in the different tasks similarly to controls, being significantly different from strongly expressing-dnSNARE mice in the four tasks (Supporting Information Figure S6a-d, left panels). It is noteworthy that the levels of expression in double-transgenic mice strongly correlate with a worse performance in the tests that rely on the dHIP-mPFC link (Supporting Information Figure S6a-d right panels), supporting the use of dnSNARE mice for in vivo testing. This means that, the higher the expression of truncated synaptobrevin II by astrocytes in vivo, the stronger the cognitive impairment. 


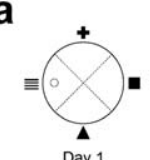

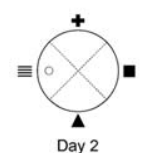

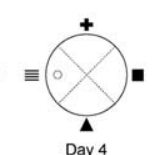
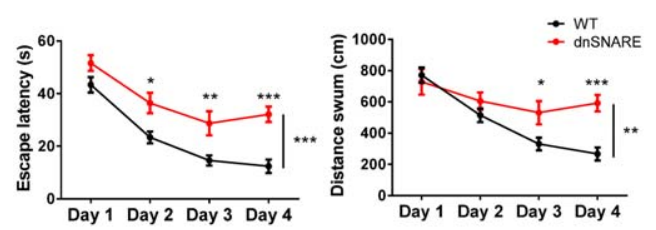

d
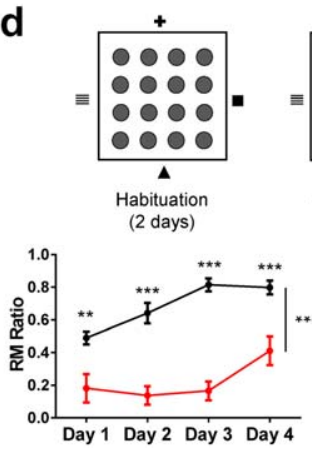

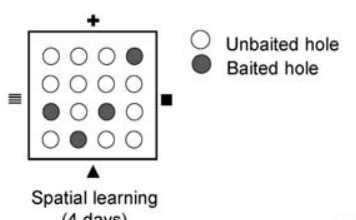
(4 days)
1.01 larning

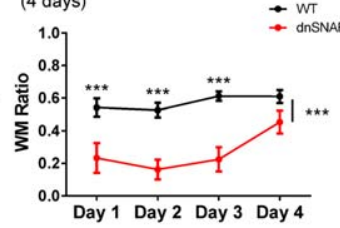

b

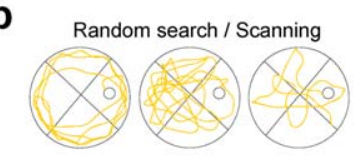

WT

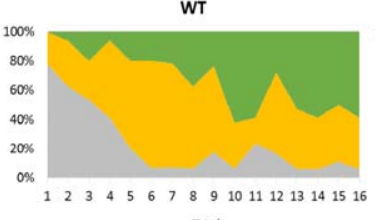

Trial

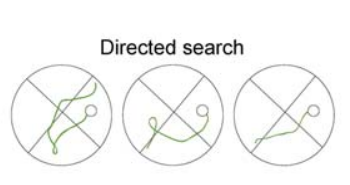

dnSNARE

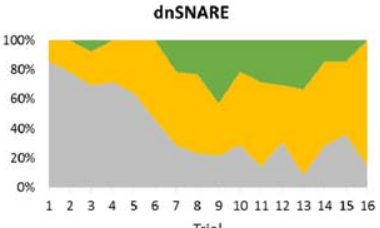

Trial
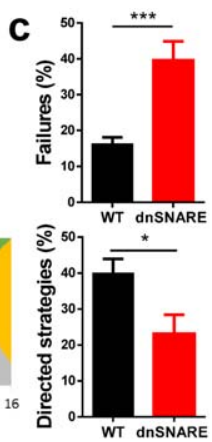

e

Failure Random Direct
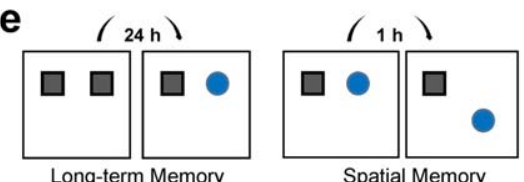

f

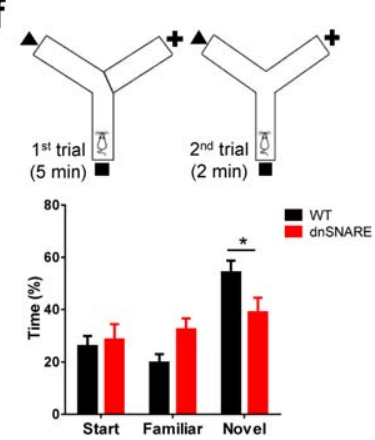

FIGURE 3 Gliotransmission impairment compromises spatial learning and reference memory in dnSNARE mice. (a-c) Morris Water Maze test ( $n=14-15$ per group); (a) reference memory task scheme (RM, top) and learning curves (bottom, two-way ANOVA repeated measures, Sidak post hoc tests) showing escape latencies and distances swam; (b) representative swim tracks used to reach the hidden platform (top), of random search/scanning (yellow) and directed search strategies (green); stacked area charts (bottom) displaying the percentage of failures (gray) and strategies used to reach platform over the 16 trials of RM for wild-type (WT, left) and dnSNARE mice (right); (c) percentage of failures (top) and cuebased/directed strategies to reach the platform in RM (bottom, $t$ tests); (d) hole board task scheme (top; baited holes, gray; unbaited holes, white); RM and working memory (WM) ratios for WT and dnSNARE mice (bottom; $n=9-14$ per group, two-way ANOVA repeated measures, Sidak post hoc tests); RM ratio: number of visits to the baited holes/total number of visits; WM ratio: number of rewarded visits/number of visits to the baited holes; (e) novel object recognition task schemes (top) for assessment of long-term (left) and spatial (right) recognition memories; percentages of exploration time of novel (bottom left) and displaced objects (bottom right; $n=7-9$ per group, $t$ test); (f) Two-trial place recognition task scheme (top); percentage of time spent in the distal third of start, familiar or novel arms in the retrieval trial (bottom; $n=9-12$ per group, two-way ANOVA, Fisher's LSD tests). WT mice represented in black and dnSNARE mice in red lines and bars. Data plotted as mean \pm SEM ${ }^{*} p<.05,{ }^{* *} p<.01,{ }^{* * *} p<.001$ [Color figure can be viewed at wileyonlinelibrary.com]

Until now, our results suggest a link between hippocampal-prefrontal synchronization impairment and poor performance in cognitive tasks that rely on those areas. To confirm this idea, we measured dHIP-mPFC synchrony in mice that performed the MWM task, and we correlated the levels of dHIP-mPFC synchronization with behavior measures. Data show that the lower levels of theta coherence directly correlate with the poor performance after the third day of the RM task (longer latencies and distances swam), with the use of directed strategies and with the success to reach the platform (Figure $4 a-c)$. Moreover, this effect was quite specific, since the dHIP-mPFC coherence in the beta band only seems to be significantly correlated with the distance swam in day 1 or the success to reach the platform (Figure $4 d-f$ ), and low gamma coherence fails to correlate with any of the parameters measured (Figure $4 \mathrm{~g}-\mathrm{i}$ ). We and others showed previously that the HIP-PFC theta coherence is crucial for reference memory processing (Benchenane et al., 2010; Jones \& Wilson, 2005; Oliveira et al., 2013; Siapas et al., 2005). Now, we specify that by releasing signaling molecules, astrocytes are required to support hippocampal-prefrontal synchronization that underlies cognitive performance.

\subsection{Neuronal structures in the dorsal hippocampus and prefrontal cortex of dnSNARE mice are intact}

The results observed hitherto prompted us to seek for structural alterations in neuronal networks that, together with the lack of astrocytic signaling, could justify the loss of theta synchronization and cognitive function observed. Therefore, we characterized dendritic morphology and spine distribution of hippocampal neurons (Lima et al., 2014) since these features may condition the operation of hippocampal networks. Tridimensional reconstruction of pyramidal neurons in the dorsal CA1 (dCA1) subregion showed that WT neurons are very similar to dnSNARE neurons (Figure 5a) for all parameters analyzed (total dendritic length, number of nodes and endings and complexity given by the number of intersections at increasing radial distances from the soma; Figure $5 b)$. Furthermore, the proximal and distal spine densities, as well as its distribution according to maturity criteria remained constant in mice of both genotypes (Figure 5c). Additional analysis of the dentate gyrus (DG) subregion of the dorsal hippocampus (Supporting Information Figure $\mathrm{S} 7 \mathrm{a}-\mathrm{c}$ ) and the prelimbic region of the prefrontal cortex (Supporting Information Figure S7d-f) showed that neuronal 
a

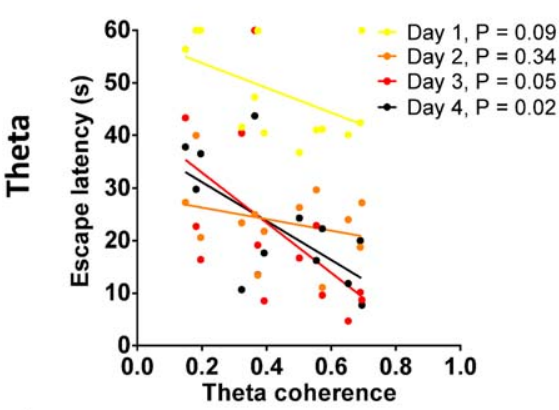

d

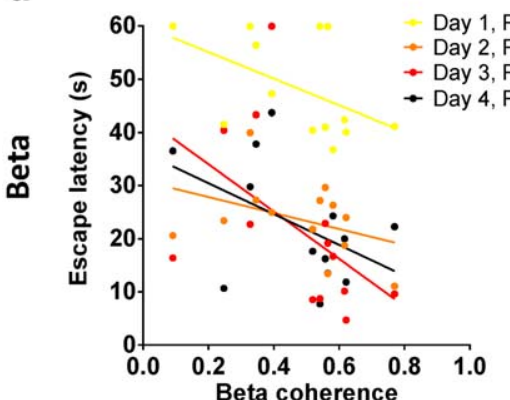

g

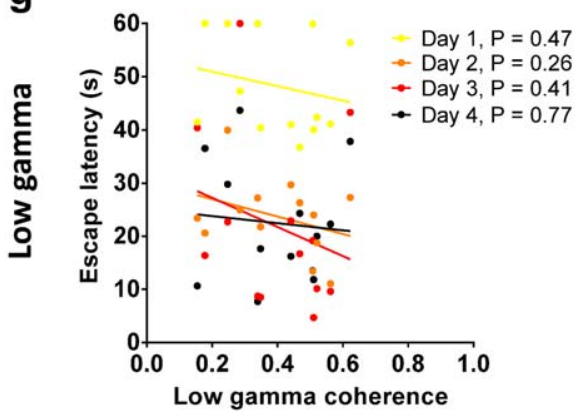

b

Distance swam

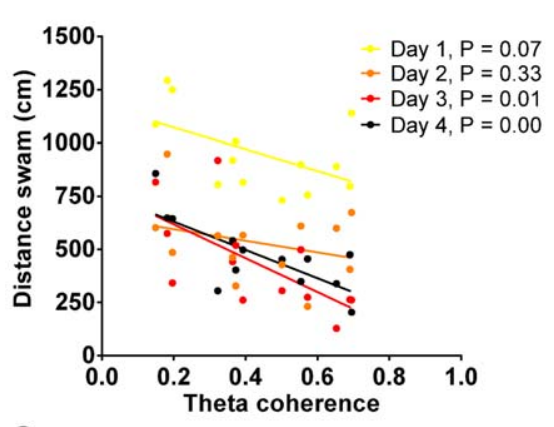

e
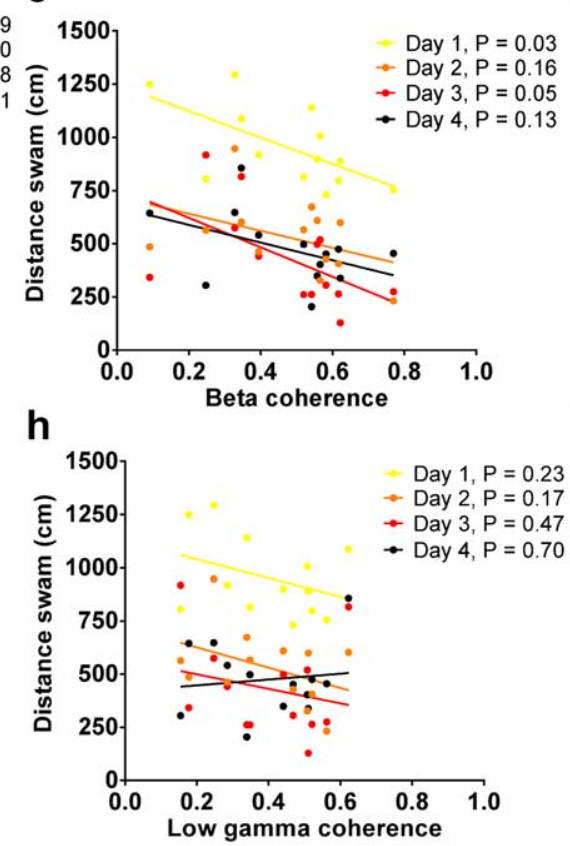

C Strategy used

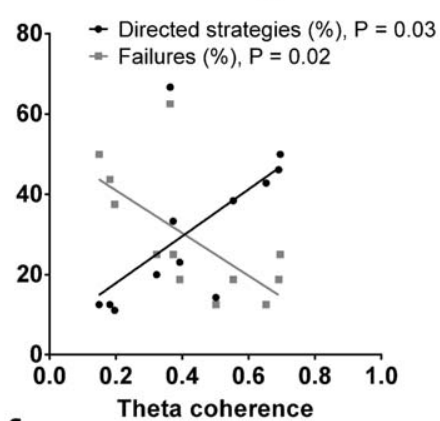

f

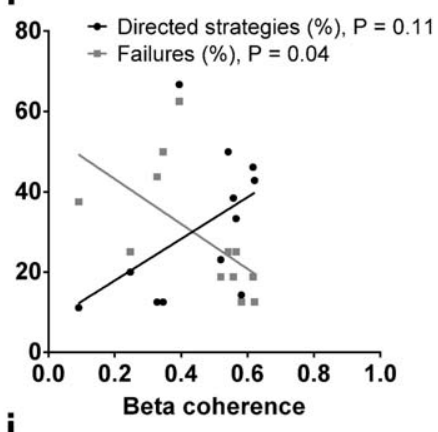

i

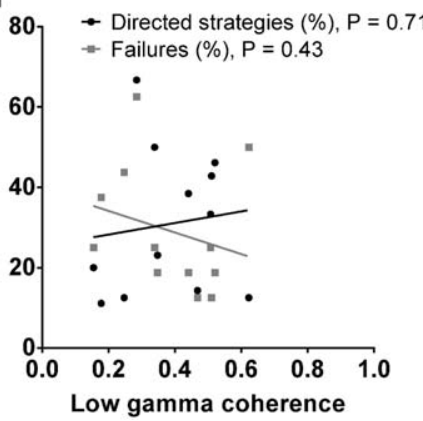

FIGURE 4 Theta dHIP-mPFC coherence correlates with the consolidation of reference memory. Pearson's correlation plots between behavior parameters analyzed for the reference memory MWM task (escape latency, distance swam, directed strategies, and failures to reach the platform) and levels of dHIP-mPFC coherence measured in the same mice (one dot per each WT and dnSNARE mouse) (a-c) theta; $(\mathrm{d}-\mathrm{f})$ beta and $(\mathrm{g}-\mathrm{i})$ low gamma frequency bands, and the respective $p$ values of each Pearson correlation. Performance of day 1 in yellow, day 2 in orange, day 3 in red, day 4 in black. The percentage of use of directed strategies is plotted in black and percentage of failures to reach platform is plotted in gray [Color figure can be viewed at wileyonlinelibrary.com]

morphologies are also intact in dnSNARE mice. It is noteworthy that our analysis covers the complete structure of the neurons, regardless of their maturity and location within the territory of transgeneexpressing astrocytes. Previously, Sultan and colleagues elegantly described changes in the morphological properties of the subset of adult-born granule neurons in the DG of dnSNARE mice, which does not collide with our data. Notably, they also showed that the existing large population of granule cells is intact. Although we cannot exclude that the sub-set of novel cells in the DG might account for the performance of the behavior tasks, the outputs of the hippocampus are produced by the majority of intact neuronal populations. Moreover, our LFP recordings are obtained by electrodes implanted in the pyramidal/radiatum layers of the CA1, where no neurogenesis occurs. Therefore, these results support the general integrity of the neuronal structures notwithstanding the lack of surrounding gliotransmitters.

\subsection{D-serine administration rescues the hippocampal-prefrontal theta coherence and the cognitive deficits in dnSNARE mice}

We next sought for the molecular mechanism of the alterations so far reported. Recent literature showed that the release of gliotransmitters is significantly decreased in the dnSNARE mice (Cao et al., 2013; Pankratov \& Lalo, 2015; Sultan et al., 2015). Amongst the different transmitters, the NMDA-receptor co-agonist D-serine arises as a good candidate, since astrocytes derived from dnSNARE mice release significantly less D-serine (Sultan et al., 2015), and D-serine is involved in synaptic modulation in the hippocampus and cortex (Fossat et al., 2012; Henneberger et al., 2010; Takata et al., 2011; Yang et al., 2003). To test whether D-serine was implicated in the dnSNARE loss of dHIPmPFC theta synchrony, and consequent cognitive deficits, we repeated these experiments including two sets of WT and dnSNARE mice 
a



dnSNARE

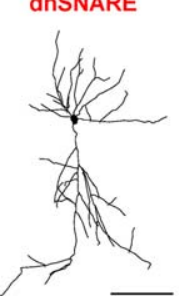

b

Pyramidal neuron morphology
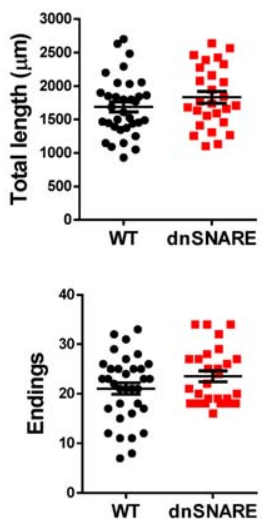


Distance from soma $(\mu \mathrm{m})$
C

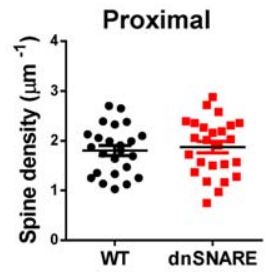

Spines distribution

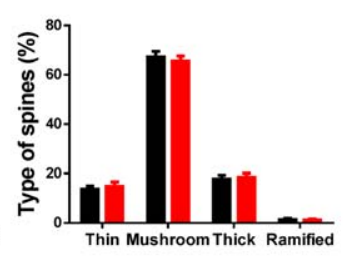

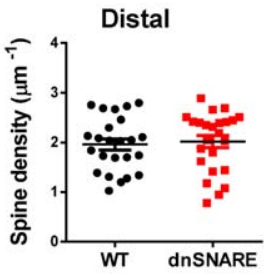

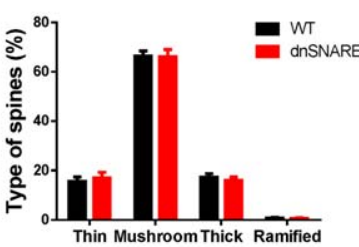

FIGURE 5 Structural characterization of neurons in the dorsal hippocampus CA1 of the WT and dnSNARE mice. (a) Representative 3D reconstructions of dorsal CA1 pyramidal neurons of WT (top) and dnSNARE mice (bottom); scale bars = $100 \mu \mathrm{m}$; (b) characterization of the $3 \mathrm{D}$ structure of apical dendrites by analysis of total dendritic length, number of nodes and endings ( $t$ test), and Sholl intersections ( $n=27-$ 34 neurons; 5 mice per group; two-way ANOVA repeated measures, Sidak post hoc tests); (c) density of spines (top) and distribution of spine types (bottom) at proximal and distal portions of the apical dendrites ( $n=19-25$ neurons; 5 mice per group; $t$ test and two-way ANOVA, Fisher's LSD tests). Data plotted as mean \pm SEM [Color figure can be viewed at wileyonlinelibrary.com]

supplemented with either saline or D-serine intraperitoneally (an approach described to increase the intracerebral levels of D-serine (Guercio et al., 2014; Han et al., 2015; Takata et al., 2011)).

First, we performed the simultaneous recording of LFPs from the dHIP and mPFC of WT and dnSNARE mice, and measured the levels of coherence between both regions longitudinally, after the administration of D-serine (Figure 6a). D-serine administration triggered a sustained increase of dHIP-mPFC theta coherence after 20-30 min to levels similar to those recorded from of WT mice in the same conditions, and significantly larger than at baseline (Figure 6a-c). Regarding beta coherence, no differences were observed between genotypes. Low gamma coherence decreased in WT mice during the protocol, however dnSNARE mice displayed levels of coherence similar to WT before and after D-serine administration. In sum, D-serine administration restored specifically the theta coherence (Figure 6d-e). Spectral power increases in the three frequency bands analyzed after the administration of D-serine regardless of the mouse genotype (Supporting Information Figure S8). Despite these results, the specific rescue of $\mathrm{dHIP}-\mathrm{mPFC}$ coherence in the theta frequency band only in the dnSNARE mice (while WT mice display stable levels of coherence after D-serine administration; Figure 6), confirms the strength of spectral coherence as a robust readout of inter-regional synchrony, independently of the amplitude of neuronal oscillations recorded.

Next, the question was if the rescue of the dHIP-mPFC theta synchronization by $\mathrm{D}$-serine supplementation would also revert the cognitive impairments observed in dnSNARE mice. Indeed, D-serine administration rescued this phenotype in the MWM test in dnSNARE mice (Figure 7a), without affecting the performance of WT mice. This is supported by the shorter latencies and distances swam, the low percentage of failures and use of directed strategies to reach the hidden platform of D-serine-treated dnSNARE mice (Figure 7b,c). In accordance, D-serine administration restored long-term recognition memory in NOR test for the dnSNARE mice to levels of WT (Figure 7d,e). It is noteworthy that both low and high transgene "expressors" preformed at levels of WT mice in the two tasks, as confirmed by the lack of correlation between GFP levels in the hippocampi of these mice and their respective behavior performance (Supporting Information Figure S9). This suggests that exogenous D-serine fulfilled the network signaling needs, despite the variable extracellular levels due to the different levels of exocytosis blockade.

Together, these results show that astrocyte-derived signaling is required for hippocampal-prefrontal theta coherence, which in turn seems to support spatial learning and reference memory. Additionally, they suggest that $\mathrm{D}$-serine might be the gliotransmitter involved in the astrocyte-neuron signaling.

\section{4 | DISCUSSION}

The use of complementary electrophysiological and behavior analysis of the dnSNARE mice to assess the involvement of astrocyte-derived gliotransmitters on cognition, suggests that the D-serine mediated signaling is critical for the synchronization of theta oscillations between the hippocampus and the prefrontal cortex, which underlies performance in spatial learning and memory tasks.

Astrocyte signaling appears to be important for the modulation of the cortical oscillations (Deng, Terunuma, Fellin, Moss, \& Haydon, 2011; Fellin et al., 2009; Lee et al., 2014; Poskanzer \& Yuste, 2016). However, the modulation of neuronal oscillations that allow the entrainment of deep brain areas required for cognitive computation remains elusive. The involvement of $\mathrm{D}$-serine mediated astrocytic 
a


b

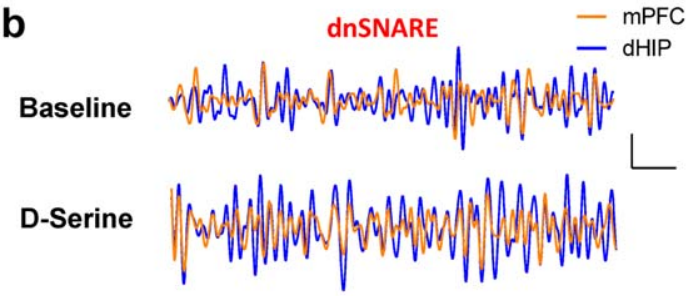

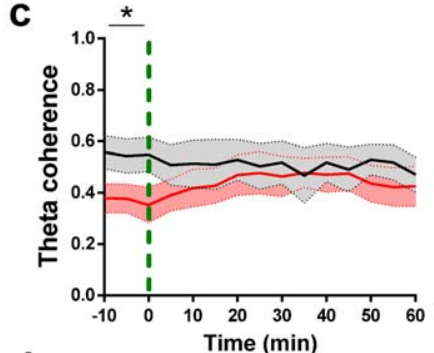
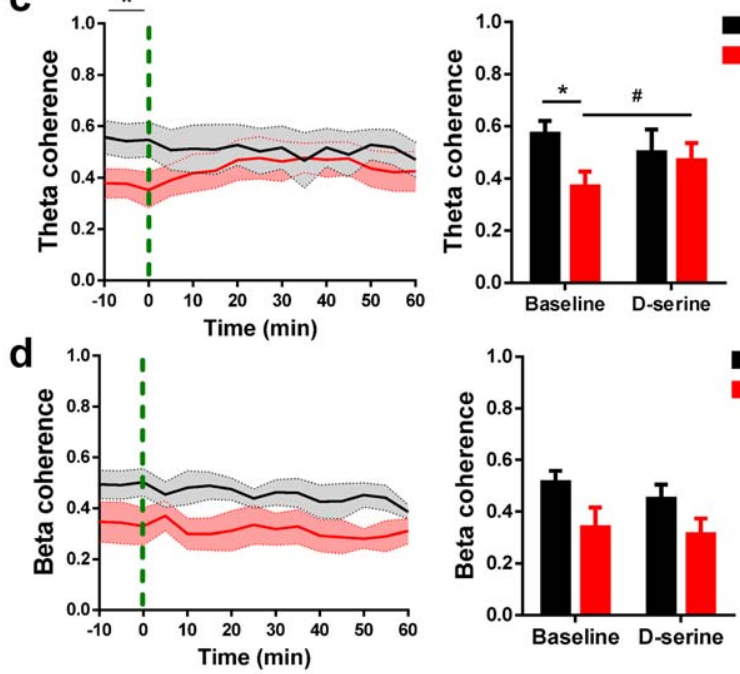

e

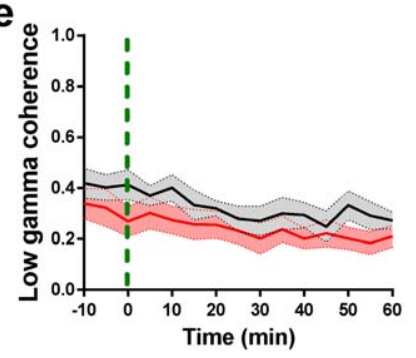

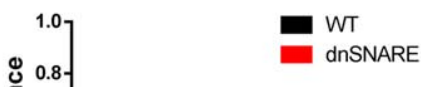

WT dnSNARE<smiles>[Tl]</smiles>
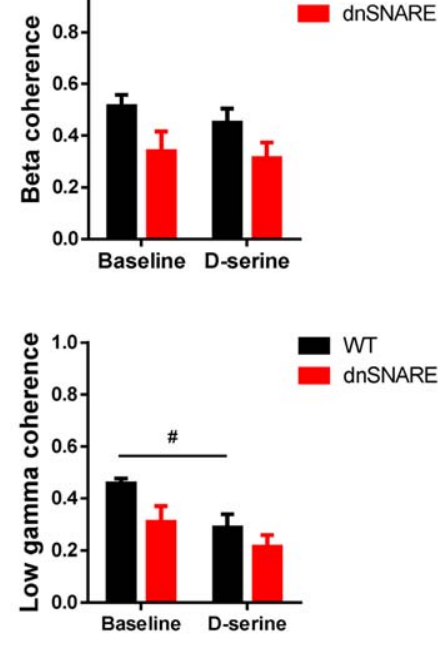

FIGURE 6 D-serine supplementation restores dorsal hippocampus-prefrontal theta synchronization in dnSNARE mice. (a) Representative heatmaps of dorsal hippocampus-prefrontal (dHIP-mPFC) spectral coherence for WT (top) and dnSNARE mice (bottom) over time; dashed line indicates the moment of D-serine administration; each spectrogram represents the dHIP-mPFC coherence calculated in intervals of $5 \mathrm{~min}$ for 4-40 Hz (theta, 4-12 Hz; beta, 12-20 Hz; low gamma, 20-40 Hz); color range: 0, dark blue; 1, red; (b) Representative theta filtered LFP traces of mPFC (orange) and dHIP (blue), recorded from dnSNARE mice at baseline or after D-serine administration (scale bars: $50 \mu \mathrm{V}, 500 \mathrm{~ms}$ ); (c-e) evolution of dHIP-mPFC coherence for theta (c), beta (d), and low gamma (e) frequencies in WT and dnSNARE mice over time (left; dashed line: administration of D-serine); values of dHIP-mPFC coherence at baseline (average of three recordings before injection) and after D-serine administration (average of three recordings, $30 \mathrm{~min}$ after injection) (right). WT mice are represented in black and dnSNARE mice in red lines and bars. Data plotted as mean \pm SEM. Two-way ANOVA, Fisher's LSD tests, ${ }^{*} p<.05$ from WT; ${ }^{*} p<.05$ from baseline ( $n=6-7$ per group) [Color figure can be viewed at wileyonlinelibrary.com]

signaling in theta HIP-PFC synchronization is supported by several pieces of correlational evidence. Theta desynchronization was observed in dnSNARE mice that display blocked exocytosis specifically in astrocytes. The expression of dnSNARE transgenes that interfere with the SNARE-complex (Bohmbach, Schwarz, Schoch, \& Henneberger, n.d.; Schubert, Bouvier, and Volterra, 2011) is restricted to astrocytes, as confirmed by: the bushy morphology of all GFP-labeled structures; the double immunostainings of the GFP reporter and cellular markers performed repeatedly by us and others (Fellin et al., 2009; Sultan et al., 2015); and the similarity between electrophysiological power $(4-40 \mathrm{~Hz})$ measured in the dHIP and mPFC of WT and dnSNARE mice discarding a neuronal compromise. Moreover, this expression of dnSNARE transgenes drastically reduced the number of fusion events and release of Dserine in astrocytes derived from dnSNARE mice (Sultan et al., 2015), leading to decreased levels of extracellular D-serine in the hippocampus and cortex of dnSNARE mice (Pankratov \& Lalo, 2015; Sultan et al., 2015). Finally, it is now well documented that astrocytic $D$-serine is critical for the function of individual synapses of the hippocampus and cortex, namely by supporting synaptic plasticity (Fossat et al., 2012; Henneberger et al., 2010; Takata et al., 2011; Yang et al., 2003).

The role of astrocytes in the modulation of neuronal ensembles is still poorly understood. The activity produced by synchronized cell ensembles generates oscillations that may vary in a wide range of frequencies. Neural oscillations are critical for information processing, and provide the nervous system with a mechanism that allow dynamic coupling within and between brain regions (Buzsáki, 2004). Theta oscillations play a pivotal role in the function of the hippocampus and in its association to cortical regions. They are a result of a concerted work of both rhythm generators and oscillators in different locations (Buzsáki, 2002). Cholinergic and GABAergic, as well as the recently described glutamatergic (Huh, Goutagny, \& Williams, 2010) afferents originating from the medial septum-diagonal band of Broca, provide the main extrinsic rhythm inputs to support theta oscillations, by modulation of interneurons, and CA1 and CA3 pyramidal neurons. Input originating from CA3 and entorhinal cortex add up to the establishment of the CA1 theta rhythm (Buzsáki, 2002). In spite of being classically accepted 
a
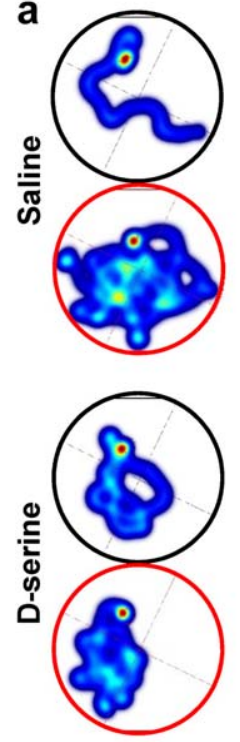

b
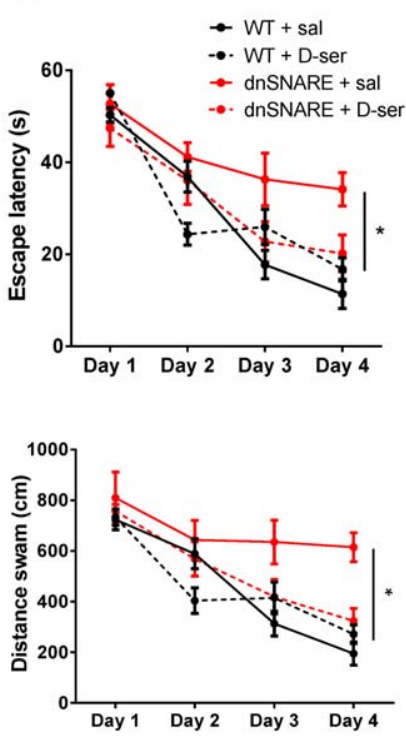

C
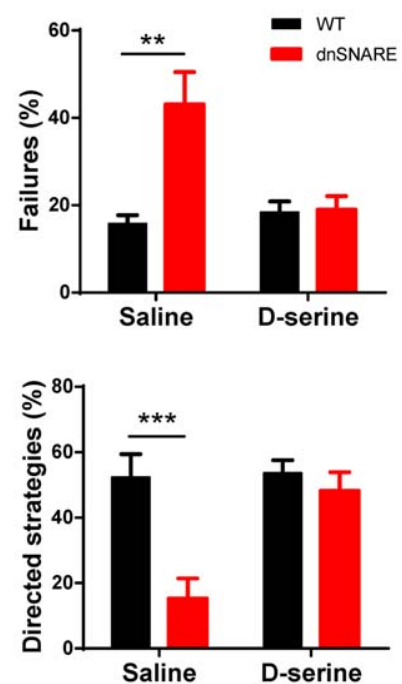

d
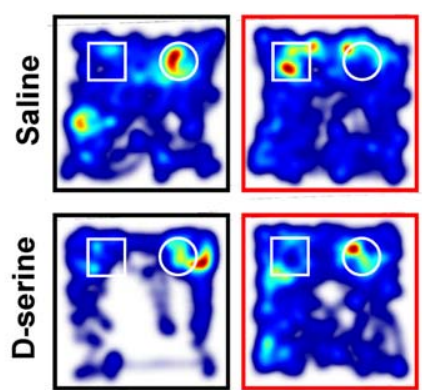

e

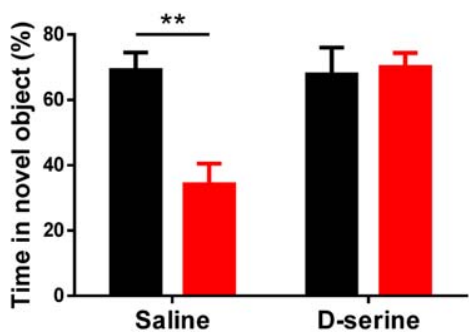

FIGURE 7 D-serine supplementation rescues the cognitive deficits observed in dnSNARE mice. (a-c) Reference memory rescue in the MWM ( $n=6-9$ per group); (a) Representative heatmaps of cumulative trajectories at day 4 of WT (black pool) and dnSNARE mice (red pool) under saline (top) and D-serine administration (bottom); (b) escape latencies (top) and distances swam (bottom) to reach the hidden platform after administration of saline (line) or D-serine (dashed line; two-way ANOVA repeated measures, Sidak post hoc tests); (c) failures to reach the platform (top) and the percentage of directed strategies used to find the platform (bottom), after administration of saline or D-serine (two-way ANOVA, Fisher's LSD tests); ( $d$-e) Long-term memory rescue in NOR ( $n=6-8$ per group); (d) representative heatmaps of cumulative exploration of a familiar (square) and novel (circle) objects in the long-term memory task, for WT (black arenas) and dnSNARE mice (red arenas), after saline (top) or D-serine administration (bottom); (e) percentages of exploration time of novel object by WT and dnSNARE, after saline or D-serine administration (two-way ANOVA, Fisher's LSD tests). WT mice are represented in black and dnSNARE mice in red lines and bars. Data plotted as mean \pm SEM. ${ }^{*} p<.05,{ }^{* *} p<.01,{ }^{* * *} p<.001$ [Color figure can be viewed at wileyonlinelibrary.com]

as oscillators, CA1 pyramidal neurons are also included in an intrinsic loop circuit, which is able to self-generate theta rhythm (Goutagny, Jackson, \& Williams, 2009). In these septal-entorhinal-hippocampal circuits, glutamatergic modulation via NMDA receptors appears to mediate theta oscillations, at least partially. Glutamatergic neurons play a role in hippocampal theta generation through local modulation of septal neurons (Robinson et al., 2016). Moreover, the pharmacological inhibition of NMDA receptors significantly impairs hippocampal theta oscillations in vivo (Lazarewicz et al., 2009), while the paired activation of NMDA and GABA receptors are able to generate theta oscillations in vitro (Kazmierska \& Konopacki, 2013). More importantly, the genetic deletion of NMDA receptors in parvalbumin-positive interneurons interfered with theta oscillations, decreasing theta-gamma phase locking, with impact in spatial memory (Korotkova, Fuchs, Ponomarenko, von Engelhardt, \& Monyer, 2010). Finally, a transgenic mouse line lacking the NR1 NMDAR subunit fails to reshape hippocampal theta rhythms after the switch between place and sequence representations in a spatial navigation task (Cabral et al., 2014). These studies denote the importance of NMDA-mediated theta oscillations for behavior performance. Due to their ubiquitous distribution and close apposition of processes endowed with machinery to send and interpret physiological signals resulting in modulation of hippocampal NMDA receptors (for review, Araque et al., 2014; Henneberger et al., 2010), astrocytes are in a good position to modulate theta oscillations. Despite the lack of literature in this sub-field, recent suggest that the physiological activation of astrocytes leads to the release of signals that may add up to hippocampal theta. The deletion of GABA B receptors specifically in astrocytes leads to a decrease in hippocampal theta oscillations (Perea et al., 2016). Moreover, septohippocampal cholinergic input to hilar astrocytes leads to hilar interneuron activation and consecutive dentate gyrus inhibition (Pabst et al., 2016), while cholinergic input to CA1 astrocytes leads to release of D-serine and modulation of CA1 pyramidal neuron activation (Papouin et al., 2017).

While the literature provides substantial amount of information on the generation of hippocampal theta oscillations, the same is not true for the understanding of synchronization of theta between the hippocampus and other brain regions, such as the cortex (Lisman \& Jensen, 2013). In our study, dnSNARE mice exhibit lower levels of dHIP-mPFC theta synchronization, whereas the two regions produce similar amounts of theta oscillations of WT controls. Since coherence measures the synchrony of both phase and amplitude of the signals, one might speculate that astrocyte modulation of NMDA receptors might have a role on the phase component of the theta produced, which could be synchronized by supplementing $D$-serine. Further experiments are needed to specifically address this issue. Together, these correlational evidences support the hypothesis that D-serine released by astrocytes modulates specifically basal theta synchronization, that may entrain unit or ensemble firing in anatomically connected cortical areas (Anderson et al., 2009; Benchenane et al., 2010; Hoffmann et al., 2015; Jones \& Wilson, 2005; O'Neill et al., 2013; Siapas et al., 2005; Yang 
et al., 2003). Nevertheless, due to the lack of literature and causal observations that support this hypothesis, further work is needed to address these open questions: (1) Does astrocyte signaling modulate simultaneously several ensembles of the septal-entorhinal-hippocampal circuit, i.e., is there a spatial modulation of the theta generation or its phase? (2) Since the administration of D-serine reaches virtually both hippocampus and prefrontal cortex indiscriminately, does HIP-PFC synchrony require simultaneous modulation in the two regions? or Does this modulation occurs in the hippocampus and from there it entrains the prefrontal cortex? (3) Since this basal HIP-PFC theta synchrony is continuously present in WT mice, does this astrocytic modulation result from a tonic release of signals that maintain it over time? How tight is the relationship between surrounding activity and exocytotic release of signals (Vardjan, Parpura, \& Zorec, 2016)?

The cognitive deficits observed in dnSNARE mice are likely a consequence of the loss of HIP-PFC synchrony, as D-serine supplementation also restored behavioral function in dnSNARE mice. However, and despite the specificity of basal desynchronization for theta frequency range in this link, we should not exclude the possibility that the hippocampus may be desynchronized with other brain regions, which could help to explain the cognitive deficits observed. Nevertheless, the basal hippocampus-prefrontal desynchronization shall be responsible for a large extent of this effect. The dorsal hippocampus is connected to prefrontal cortex indirectly. The loop closes by a multi-synaptic circuit, being the prefrontal cortex connected to the hippocampus also indirectly (Preston \& Eichenbaum, 2013). Within this circuit, the dorsal hippocampus performs primarily cognitive functions, while the ventral relates to emotional behavior (Fanselow \& Dong, 2010). Moreover, synchrony between oscillations of hippocampal dorsal or ventral subregions, and PFC has been related respectively to cognitive (O'Neill et al., 2013) and emotional behavior (Adhikari, Topiwala, \& Gordon, 2010; Kafetzopoulos et al., 2017; Mateus-Pinheiro et al., 2016; Oliveira et al., 2013). Specifically, and despite the indirect connections, O'Neill and colleagues demonstrated that dHIP theta oscillations are synchronized with theta oscillations in the mPFC, and that theta synchrony is critical for a spatial memory task. Interestingly, both single units and ensembles in the prefrontal cortex appear to entrain with the theta rhythm from the dorsal hippocampus. On the one hand, this type of memory task was previously shown to be dependent on the synchronization of theta oscillations between the hippocampus and prefrontal cortex (Benchenane et al., 2010; Jones \& Wilson, 2005; Siapas et al., 2005). Our data shows now that the poor consolidation of reference memory directly correlates with lower levels of basal theta coherence presented by a given dnSNARE mouse, which is in accordance with previous reports showing that the pathological decrease of theta coherence triggered this cognitive deficit (Gordon, 2011; Oliveira et al., 2013). On the other hand, the higher expression of dnSNARE transgenes in the hippocampus strongly correlates with a worse cognitive performance in doubletransgenic mice, suggesting that those impairments are due to the lack of astrocyte-derived exocytosis. It is noteworthy, that this variability of genetic modulation may explain negative results in studies in which mice displaying variable levels of transgene expression or genomic recombination were analyzed as a single group (Oliveira et al., 2015).
In conclusion, our results provide the first evidence of a mechanism by which $D$-serine mediated astrocytic signaling is required for entrainment of distant corticolimbic circuits, being mandatory for cognitive performance.

\section{ACKNOWLEDGMENT}

The authors are grateful to Prof. Philip Haydon for sharing the mice lines and for the valuable comments on this manuscript. We also acknowledge the technical support of Goreti Pinto, and the help of Ana Lima, Ana Filipa Oliveira and Anabela Fernandes on colony implementation and management. The authors acknowledge funding from national funds through Foundation for Science and Technology (FCT) project (PTDC/SAU-NSC/118194/2010) to V.M.S., S.G.G., G. T., M.M., J.S.R., J.S.C., J.F.O. and fellowships (SFRH/BD/89714/ 2012 to V.M.S., SFRH/BPD/97281/2013 to J.F.O., SFRH/BD/ $101298 / 2014$ to S.G.G., IF/00328/2015 to JFO, IF/01079/2014 to LP); Marie Curie Fellowship FP7-PEOPLE-2010-IEF 273936 and BIAL Foundation Grants 207/14 to J.F.O. and 427/14 to LP; Northern Portugal Regional Operational Programme (NORTE 2020), under the Portugal 2020 Partnership Agreement, through the European Regional Development Fund (FEDER)(NORTE-01-0145-FEDER000013); FEDER funds, through the Competitiveness Factors Operational Programme (COMPETE), and National funds, through the FCT (POCl-01-0145-FEDER-007038).

\section{CONFLICTS OF INTEREST}

The authors declare no competing financial interests.

\section{ORCID}

João Filipe Oliveira (D) http://orcid.org/0000-0002-1005-2328

\section{REFERENCES}

Adhikari, A., Topiwala, M. A., \& Gordon, J. A. (2010). Synchronized activity between the ventral hippocampus and the medial prefrontal cortex during anxiety. Neuron, 65, 257-269.

Anderson, K. L., Rajagovindan, R., Ghacibeh, G. A., Meador, K. J., \& Ding, M. (2009). Theta oscillations mediate interaction between prefrontal cortex and medial temporal lobe in human memory. Cerebral Cortex, 20, 1604-1612.

Araque, A., Carmignoto, G., Haydon, P. G., Oliet, S. H. R., Robitaille, R., \& Volterra, A. (2014). Gliotransmitters travel in time and space. Neuron, $81,728-739$

Bail, M. L., Martineau, M., Sacchi, S., Yatsenko, N., Radzishevsky, I., Conrod, S., ... Mothet, J.-P. (2015). Identity of the NMDA receptor coagonist is synapse specific and developmentally regulated in the hippocampus. Proceedings of the National Academy of Sciences, 112, E204-E213.

Balu, D. T., \& Coyle, J. T. (2012). Neuronal d-serine regulates dendritic architecture in the somatosensory cortex. Neuroscience Letters, 517, 77-81.

Benchenane, K., Peyrache, A., Khamassi, M., Tierney, P. L., Gioanni, Y., Battaglia, F. P., \& Wiener, S. I. (2010). Coherent theta oscillations and reorganization of spike timing in the hippocampal-prefrontal network upon learning. Neuron, 66, 921-936. 
Bohmbach, K., Schwarz, M. K., Schoch, S., \& Henneberger, C. (2017). The structural and functional evidence for vesicular release from astrocytes in situ. Brain Research Bulletin. doi:10.1016/j. brainresbull.2017.01.015

Buzsáki, G. (2002). Theta oscillations in the hippocampus. Neuron, 33, 325-340.

Buzsáki, G. (2004). Large-scale recording of neuronal ensembles. Nature Neurosciences, 7, 446-451.

Cabral, H. O., Vinck, M., Fouquet, C., Pennartz, C. M. A., Rondi-Reig, L., \& Battaglia, F. P. (2014). Oscillatory dynamics and place field maps reflect hippocampal ensemble processing of sequence and place memory under NMDA receptor control. Neuron, 81, 402-415.

Cao, X., Li, L.-P., Wang, Q., Wu, Q., Hu, H.-H., Zhang, M., ... Gao, T.-M. (2013). Astrocyte-derived ATP modulates depressive-like behaviors. Nature Medicine, 19, 773-777.

Castilla-Ortega, E., Sánchez-López, J., Hoyo-Becerra, C., Matas-Rico, E., Zambrana-Infantes, E., Chun, J., ... Santin, L. J. (2010). Exploratory, anxiety and spatial memory impairments are dissociated in mice lacking the LPA1 receptor. Neurobiology of Learning \& Memory, 94, 7382.

Clarke, L. E., \& Barres, B. A. (2013). Emerging roles of astrocytes in neural circuit development. Nature Reviews: Neuroscience, 14, 311-321.

Costa-Aze, V. D. S., Quiedeville, A., Boulouard, M., \& Dauphin, F. (2012). 5-HT6 receptor blockade differentially affects scopolamine-induced deficits of working memory, recognition memory and aversive learning in mice. Psychopharmacology, 222, 99-115.

Deng, Q., Terunuma, M., Fellin, T., Moss, S. J., \& Haydon, P. G. (2011). Astrocytic activation of $\mathrm{A} 1$ receptors regulates the surface expression of NMDA receptors through a Src kinase dependent pathway. Glia, 59, 1084-1093.

Fanselow, M. S., \& Dong, H.-W. (2010). Are the dorsal and ventral hippocampus functionally distinct structures? Neuron, 65, 7-19.

Fellin, T., Halassa, M. M., Terunuma, M., Succol, F., Takano, H., Frank, M., ... Haydon, P. G. (2009). Endogenous nonneuronal modulators of synaptic transmission control cortical slow oscillations in vivo. Proceedings of the National Academy of Sciences of the United States of America, 106, 15037-15042.

Florian, C., Vecsey, C. G., Halassa, M. M., Haydon, P. G., \& Abel, T. (2011). Astrocyte-derived adenosine and A1 receptor activity contribute to sleep loss-induced deficits in hippocampal synaptic plasticity and memory in mice. The Journal of Neuroscience, 31, 6956-6962.

Fossat, P., Turpin, F. R., Sacchi, S., Dulong, J., Shi, T., Rivet, J.-M., ... Mothet, J.-P. (2012). Glial d-serine gates NMDA receptors at excitatory synapses in prefrontal cortex. Cerebral Cortex, 22, 595-606.

Fujita, T., Chen, M. J., Li, B., Smith, N. A., Peng, W., Sun, W., ... Nedergaard, M. (2014). Neuronal transgene expression in dominantnegative SNARE mice. Journal of Neuroscience, 34, 16594-16604.

Gordon, J. A. (2011). Oscillations and hippocampal-prefrontal synchrony. Current Opinion in Neurobiology, 21, 486-491.

Goutagny, R., Jackson, J., \& Williams, S. (2009). Self-generated theta oscillations in the hippocampus. Nature Neuroscience, 12, 1491-1493.

Graziano, A., Petrosini, L., \& Bartoletti, A. (2003). Automatic recognition of explorative strategies in the Morris water maze. Journal of Neuroscience Methods, 130, 33-44.

Guercio, G. D., Bevictori, L., Vargas-Lopes, C., Madeira, C., Oliveira, A., Carvalho, V. F., ... Panizzutti, R. (2014). d-Serine prevents cognitive deficits induced by acute stress. Neuropharmacology, 86, 1-8.

Halassa, M. M., Florian, C., Fellin, T., Munoz, J. R., Lee, S.-Y., Abel, T., ... Frank, M. G. (2009). Astrocytic modulation of sleep homeostasis and cognitive consequences of sleep loss. Neuron, 61, 213-219.
Han, H., Peng, Y., \& Dong, Z. (2015). d-Serine rescues the deficits of hippocampal long-term potentiation and learning and memory induced by sodium fluoroacetate. Pharmacology Biochemistry \& Behavior, 133, 51-56.

Haydon, P. G., \& Nedergaard, M. (2015). How do astrocytes participate in neural plasticity? Cold Spring Harbor Perspectives in Biology, 7, a020438.

Henneberger, C., Papouin, T., Oliet, S. H. R., \& Rusakov, D. A. (2010). Long-term potentiation depends on release of $\mathrm{d}$-serine from astrocytes. Nature, 463, 232-236.

Hoffmann, L. C., Cicchese, J. J., \& Berry, S. D. (2015). Harnessing the power of theta: Natural manipulations of cognitive performance during hippocampal theta-contingent eyeblink conditioning. Frontiers in Systems Neuroscience, 9. doi:10.3389/fnsys.2015.00050

Huh, C. Y. L., Goutagny, R., \& Williams, S. (2010). Glutamatergic neurons of the mouse medial septum and diagonal band of Broca synaptically drive hippocampal pyramidal cells: Relevance for hippocampal theta rhythm. Journal of Neuroscience, 30, 15951-15961.

Jones, M. W., \& Wilson, M. A. (2005). Theta rhythms coordinate hippocampal-prefrontal interactions in a spatial memory task. PLoS Biology, 3, e402.

Kafetzopoulos, V., Kokras, N., Sotiropoulos, I., Oliveira, J. F., LeiteAlmeida, H., Vasalou, A., ... Dalla, C. (2017). The nucleus reuniens: A key node in the neurocircuitry of stress and depression. Molecular Psychiatry. https://doi.org/10.1038/mp.2017.55

Kang, N., Peng, H., Yu, Y., Stanton, P. K., Guilarte, T. R., \& Kang, J. (2013). Astrocytes release d-serine by a large vesicle. Neuroscience, 240, 243-257.

Kazmierska, P., \& Konopacki, J. (2013). Development of NMDA-induced theta rhythm in hippocampal formation slices. Brain Research Bulletin, 98, 93-101.

Khakh, B. S., \& Sofroniew, M. V. (2015). Diversity of astrocyte functions and phenotypes in neural circuits. Nature Neuroscience, 18, 942-952.

Korotkova, T., Fuchs, E. C., Ponomarenko, A., von Engelhardt, J., \& Monyer, H. (2010). NMDa receptor ablation on parvalbumin-positive interneurons impairs hippocampal synchrony, spatial representations, and working memory. Neuron, 68, 557-569.

Lazarewicz, M. T., Ehrlichman, R. S., Maxwell, C. R., Gandal, M. J., Finkel, L. H., \& Siegel, S. J. (2009). Ketamine modulates theta and gamma oscillations. Journal of Cognitive Neuroscience, 22, 1452-1464.

Lee, H. S., Ghetti, A., Pinto-Duarte, A., Wang, X., Dziewczapolski, G., Galimi, F., ... Heinemann, S. F. (2014). Astrocytes contribute to gamma oscillations and recognition memory. Proceedings of the National Academy of Sciences, 111, E3343-E3352.

Leger, M., Quiedeville, A., Bouet, V., Haelewyn, B., Boulouard, M., Schumann-Bard, P., \& Freret, T. (2013). Object recognition test in mice. Nature Protocols, 8, 2531-2537.

Lima, A., Sardinha, V. M., Oliveira, A. F., Reis, M., Mota, C., Silva, M. A., ... Oliveira, J. F. (2014). Astrocyte pathology in the prefrontal cortex impairs the cognitive function of rats. Molecular Psychiatry, 19, 834-841.

Lisman, J. E., \& Jensen, O. (2013). The theta-gamma neural code. Neuron, 77, 1002-1016.

Martineau, M., Galli, T., Baux, G., \& Mothet, J.-P. (2008). Confocal imaging and tracking of the exocytotic routes for d-serine-mediated gliotransmission. Glia, 56, 1271-1284.

Martineau, M., Shi, T., Puyal, J., Knolhoff, A. M., Dulong, J., Gasnier, B., ... Mothet, J.-P. (2013). Storage and uptake of d-serine into astrocytic synaptic-like vesicles specify gliotransmission. The Journal of Neuroscience, 33, 3413-3423.

Mateus-Pinheiro, A., Alves, N. D., Patrício, P., Machado-Santos, A. R., Loureiro-Campos, E., Silva, J. M., ... Pinto, L. (2016). AP2 $\gamma$ controls 
adult hippocampal neurogenesis and modulates cognitive, but not anxiety or depressive-like behavior. Molecular Psychiatry. https://doi. org/10.1038/mp.2016.169

Miller, R. F. (2004). d-serine as a glial modulator of nerve cells. Glia, 47, 275-283.

Mitra, P. P., \& Pesaran, B. (1999). Analysis of dynamic brain imaging data. Biophysical Journal, 76, 691-708.

Nakashiba, T., Young, J. Z., McHugh, T. J., Buhl, D. L., \& Tonegawa, S. (2008). Transgenic inhibition of synaptic transmission reveals role of CA3 output in hippocampal learning. Science (New York, NY), 319, 1260-1264

Oliet, S. H. R., \& Mothet, J.-P. (2006). Molecular determinants of dserine-mediated gliotransmission: From release to function. Glia, 54, 726-737.

Oliveira, J. F., Dias, N. S., Jacinto, L. R., Cerqueira, J. J., \& Sousa, N. (2013). Chronic stress disrupts neural coherence between corticolimbic structures. Frontiers in Neural Circuits, 7, 10.

Oliveira, J. F., Sardinha, V. M., Guerra-Gomes, S., Araque, A., \& Sousa, N. (2015). Do stars govern our actions? Astrocyte involvement in rodent behavior. Trends in Neurosciences, 38, 535-549.

O'Neill, P.-K., Gordon, J. A., \& Sigurdsson, T. (2013). Theta oscillations in the medial prefrontal cortex are modulated by spatial working memory and synchronize with the hippocampus through its ventral subregion. Journal of Neuroscience, 33, 14211-14224.

Pabst, M., Braganza, O., Dannenberg, H., Hu, W., Pothmann, L., Rosen, J., ... Beck, H. (2016). Astrocyte intermediaries of septal cholinergic modulation in the hippocampus. Neuron, 90, 853-865.

Pankratov, Y., \& Lalo, U. (2015). Role for astroglial $\alpha 1$-adrenoreceptors in gliotransmission and control of synaptic plasticity in the neocortex. Frontiers in Cellular Neuroscience, 9. https://doi.org/10.3389/fncel. 2015.00230

Pannasch, U., Freche, D., Dallérac, G., Ghézali, G., Escartin, C., Ezan, P., ... Rouach, N. (2014). Connexin 30 sets synaptic strength by controlling astroglial synapse invasion. Nature Neuroscience, 17, 549-558.

Papouin, T., Dunphy, J. M., Tolman, M., Dineley, K. T., \& Haydon, P. G. (2017). Septal cholinergic neuromodulation tunes the astrocytedependent gating of hippocampal NMDA receptors to wakefulness. Neuron, 94, 840-854.e7.

Papouin, T., Henneberger, C., Rusakov, D. A., \& Oliet, S. H. R. (2017). Astroglial versus neuronal d-serine: Fact checking. Trends in Neurosciences. https://doi.org/10.1016/j.tins.2017.05.007[]

Pascual, O., Casper, K. B., Kubera, C., Zhang, J., Revilla-Sanchez, R., Sul, J.-Y., ... Haydon, P. G. (2005). Astrocytic purinergic signaling coordinates synaptic networks. Science (New York, NY), 310, 113-116.

Paxinos, G., \& Franklin, K. B. J. (2001). The mouse brain in stereotaxic coordinates (2nd ed.). San Diego: Academic Press.

Perea, G., Gómez, R., Mederos, S., Covelo, A., Ballesteros, J. J., Schlosser, L., ... Araque, A. (2016). Activity-dependent switch of GABAergic inhibition into glutamatergic excitation in astrocyte-neuron networks. ELife, 5, e20362.

Perea, G., Sur, M., \& Araque, A. (2014). Neuron-glia networks: Integral gear of brain function. Frontiers in Cellular Neuroscience, 8, 378.

Petrelli, F., \& Bezzi, P. (2016). Novel insights into gliotransmitters. Current Opinion in Pharmacology, 26, 138-145.

Poskanzer, K. E., \& Yuste, R. (2016). Astrocytes regulate cortical state switching in vivo. Proceedings of the National Academy of Sciences, 113, E2675-E2684.

Preston, A. R., \& Eichenbaum, H. (2013). Interplay of hippocampus and prefrontal cortex in memory. Current Biology, 23, R764-R773.
Robinson, J., Manseau, F., Ducharme, G., Amilhon, B., Vigneault, E., Mestikawy, S. E., \& Williams, S. (2016). Optogenetic activation of septal glutamatergic neurons drive hippocampal theta rhythms. Journal of Neuroscience, 36, 3016-3023.

Rosenberg, D., Artoul, S., Segal, A. C., Kolodney, G., Radzishevsky, I., Dikopoltsev, E., ... Wolosker, H. (2013). Neuronal d-serine and glycine release via the Asc-1 transporter regulates NMDA receptordependent synaptic activity. Journal of Neuroscience, 33, 3533-3544.

Rusakov, D. A. (2015). Disentangling calcium-driven astrocyte physiology. Nature Reviews Neuroscience, 16, 226-233.

Schubert, V., Bouvier, D., \& Volterra, A. (2011). SNARE protein expression in synaptic terminals and astrocytes in the adult hippocampus: A comparative analysis. Glia, 59, 1472-1488.

Siapas, A. G., Lubenov, E. V., \& Wilson, M. A. (2005). Prefrontal phase locking to hippocampal theta oscillations. Neuron, 46, 141-151.

Sigurdsson, T., Stark, K. L., Karayiorgou, M., Gogos, J. A., \& Gordon, J. A. (2010). Impaired hippocampal-prefrontal synchrony in a genetic mouse model of schizophrenia. Nature, 464, 763-767.

Sultan, S., Li, L., Moss, J., Petrelli, F., Cassé, F., Gebara, E., ... Toni, N. (2015). Synaptic integration of adult-born hippocampal neurons is locally controlled by astrocytes. Neuron, 88, 957-972.

Takata, N., Mishima, T., Hisatsune, C., Nagai, T., Ebisui, E., Mikoshiba, K., \& Hirase, H. (2011). Astrocyte calcium signaling transforms cholinergic modulation to cortical plasticity in vivo. The Journal of Neuroscience, 31, 18155-18165.

Vardjan, N., Parpura, V., \& Zorec, R. (2016). Loose excitation-secretion coupling in astrocytes. Glia, 64, 655-667.

Verkhratsky, A., Matteoli, M., Parpura, V., Mothet, J.-P., \& Zorec, R. (2016). Astrocytes as secretory cells of the central nervous system: Idiosyncrasies of vesicular secretion. The EMBO Journal. 35, 239-257.

Volterra, A., Liaudet, N., \& Savtchouk, I. (2014). Astrocyte $\mathrm{Ca}^{2+}$ signalling: An unexpected complexity. Nature Reviews Neuroscience, 15, 327-335.

Wagner, L., Pannicke, T., Rupprecht, V., Frommherz, I., Volz, C., Illes, P., ... Grosche, A. (2017). Suppression of SNARE-dependent exocytosis in retinal glial cells and its effect on ischemia-induced neurodegeneration. Glia, 65, 1059-1071.

Yang, Y., Ge, W., Chen, Y., Zhang, Z., Shen, W., Wu, C., ... Duan, S. (2003). Contribution of astrocytes to hippocampal long-term potentiation through release of $\mathrm{d}$-serine. Proceedings of the National Academy of Sciences, 100, 15194-15199.

Zhan, Y. (2015). Theta frequency prefrontal-hippocampal driving relationship during free exploration in mice. Neuroscience, 300, 554565.

Zhang, Q., Pangršič, T., Kreft, M., Kržan, M., Li, N., Sul, J.-Y., ... Haydon, P. G. (2004). Fusion-related release of glutamate from astrocytes. Journal of Biological Chemistry, 279, 12724-12733.

\section{SUPPORTING INFORMATION}

Additional Supporting Information may be found online in the supporting information tab for this article.

How to cite this article: Sardinha VM, Guerra-Gomes S, Caetano I, et al. Astrocytic signaling supports hippocampal-prefrontal theta synchronization and cognitive function. Glia. 2017;65:1944-1960. https://doi.org/10.1002/glia.23205 